\title{
Recent advances in organocatalytic asymmetric aza-Michael reactions of amines and amides
}

\author{
Pratibha Sharma, Raakhi Gupta and Raj K. Bansal ${ }^{*}$
}

\author{
Review \\ Address: \\ Department of Chemistry, The IIS (deemed to be University), Jaipur \\ 302 020, India \\ Email: \\ Raj K. Bansal ${ }^{*}$ - bansal56@gmail.com \\ * Corresponding author \\ Keywords: \\ asymmetric aza-Michael reaction; covalent bonding catalysis; nitrogen \\ heterocycles; non-covalent bonding catalysis; organocatalysis
}

\author{
Beilstein J. Org. Chem. 2021, 17, 2585-2610. \\ https://doi.org/10.3762/bjoc.17.173 \\ Received: 12 July 2021 \\ Accepted: 27 September 2021 \\ Published: 18 October 2021 \\ This article is part of the thematic issue "New advances in asymmetric \\ organocatalysis".
}

Guest Editor: R. Šebesta

(C) 2021 Sharma et al.; licensee Beilstein-Institut.

License and terms: see end of document.

\begin{abstract}
Nitrogen-containing scaffolds are ubiquitous in nature and constitute an important class of building blocks in organic synthesis. The asymmetric aza-Michael reaction (aza-MR) alone or in tandem with other organic reaction(s) is an important synthetic tool to form new $\mathrm{C}-\mathrm{N}$ bond(s) leading to developing new libraries of diverse types of bioactive nitrogen compounds. The synthesis and application of a variety of organocatalysts for accomplishing highly useful organic syntheses without causing environmental pollution in compliance with "Green Chemistry" has been a landmark development in the recent past. Application of many of these organocatalysts has been extended to asymmetric aza-MR during the last two decades. The present article overviews the literature published during the last 10 years concerning the asymmetric aza-MR of amines and amides catalysed by organocatalysts. Both types of the organocatalysts, i.e., those acting through non-covalent interactions and those working through covalent bond formation have been applied for the asymmetric aza-MR. Thus, the review includes the examples wherein cinchona alkaloids, squaramides, chiral amines, phase-transfer catalysts and chiral bifunctional thioureas have been used, which activate the substrates through hydrogen bond formation. Most of these reactions are accompanied by high yields and enantiomeric excesses. On the other hand, N-heterocyclic carbenes and chiral pyrrolidine derivatives acting through covalent bond formation such as the iminium ions with the substrates have also been included. Wherever possible, a comparison has been made between the efficacies of various organocatalysts in asymmetric aza-MR.
\end{abstract}

\section{Introduction}

The Michael reaction though discovered about 135 years ago $[1,2]$ continues to attract attention of the chemists owing to its potential of making a vast variety of organic compounds partic- ularly of pharmacological importance accessible. Over the years, its many versions known as aza-Michael, thio-Michael, oxa-Michael, phospha-Michael, etc. have been developed and 
well exploited for their synthetic applications [3-7]. The reaction involving a nitrogen-based nucleophile as the Michael donor is known as the aza-Michael reaction (aza-MR). In view of its ability to introduce a nitrogen-containing functionality at the $\beta$-position of an activated alkenyl- or alkynyl-substrate, over the years, it has developed as an important synthetic strategy for the preparation of a large variety of $\beta$-amino carbonyl and similar motifs which are present in many bioactive natural products [8,9], antibiotics [10-12] and chiral auxiliaries [13-15]. However, the reaction of many nitrogen-nucleophiles, such as aromatic amines, amides, imides, etc. require the use of an appropriate catalyst to undergo a Michael addition with a suitable acceptor. In view of this, chemists endeavoured to develop different types of catalysts, particularly the chiral catalysts to accomplish asymmetric aza-MRs. The development of metal-free small organic molecules as catalysts has been a landmark advancement in organic synthesis in the recent past [16]. MacMillan and co-workers for the first time in the year 2000 termed these catalysts as 'Organocatalysts' [17]. It was followed by intense activity and phenomenal rise in the number of publications in this field. These organocatalysts have been found compatible with many aspects of 'Green Chemistry' on the one hand, and highly selective in many organic syntheses on the other hand [17]. It has an added advantage that a large number of enantiomerically pure organocatalysts can be accessed from the chiral pool. Both types of organocatalysts, namely those acting through non-covalent bonding as well as those working by making covalent bonding have been employed for accomplishing asymmetric aza-MRs.

There are several review articles available on organocatalytic asymmetric aza-MRs, each highlighting a certain aspect of the reaction. While Sánchez-Roselló et al. [18] classified these reactions on the basis of the nature of the substrates, Nayak et al. [19] and Bhanja et al. [20] focused on the stereoselective synthesis of nitrogen heterocycles via Michael cascade reactions. Recently, Vinogradov et al. [21] reviewed the synthesis of pharmacology-relevant nitrogen heterocycles via stereoselective aza-MRs. On the other hand, Enders et al. [22], Wang et al. [23] as well as Krishna et al. [24] highlighted the scope and catalytic performances of some organocatalysts in asymmetric aza-MRs. However, the last three review articles are almost 10 years old and they do not cover the application of many important organocatalysts, such as thioureas and nitrogen heterocyclic carbenes (NHCs) used for the asymmetric aza-MRs. Furthermore, in the last review article [24], the application of organocatalysts is included as a small part of a general review. In view of this, we considered it prudent to compile this mini review exclusively based on the application of all categories of the organocatalysts and highlighting their efficacies covering the literature of the last ten years.

\section{Review}

In the present review, the known stereoselective syntheses of pharmacology-oriented nitrogen containing heterocyclic scaffolds via non-covalent bonding and covalent bonding organocatalytic aza-MRs has been systematized. This classification is especially useful for researchers to understand both the noncovalent and covalent organocatalysis.

It is intended to overview the literature of the last 10 years, i.e., from 2011 through 2020 only. Nevertheless, wherever necessary, earlier references may also be cited to maintain coherence. Furthermore, nitrogen nucleophiles comprise a large variety of compounds; however, in order to comply with the requirements of a mini review, additions of amines and amides only will be included.

\section{Non-covalent bonding organocatalytic aza-Michael reactions}

Organocatalysts catalyzing aza-MRs through mainly hydrogen bonding include cinchona alkaloids, squaramide derivatives, phase-transfer catalysts and bifunctional thiourea derivatives.

\subsection{Reactions catalyzed by chiral cinchona alkaloid derivatives}

Cai et al. prepared and used a number of organocatalysts from Cinchona alkaloids for the aza-MR of aniline (1) with chalcone (2) to obtain the adducts 4 in poor to very good yields (24 to $>99 \%$ ) with poor to moderate ee ( 9 to $55 \%$ ). A complete reversal of stereoselectivity was observed on introducing a benzoyl group in cinchonine and cinchonidine. It was demonstrated that racemization occurred in suitable solvents under mild conditions due to retro-MR of the initially formed Michael adduct (Scheme 1) [25]. The proposed catalytic cycle involved generation of the active complex through hydrogen bonding between catalyst and aniline followed by interaction with chalcone via $\pi-\pi$ stacking of aromatic rings and hydrogen bonding leading to the Michael adduct.

Likewise, Lee et al. reported cinchona-based primary amine catalyzed cascade aza-Michael-aldol reaction of $\alpha, \beta$-unsaturated ketones 6 with 2-(1H-pyrrol-2-yl)-2-oxoacetates 5 where triphenylacetic acid was used as an additive. This cascade reaction afforded highly functionalized chiral pyrrolizines $\mathbf{8}$ in good yields (70-91\%) with excellent levels of stereocontrol ( $\approx 92 \%$ ee, $>20: 1 \mathrm{dr}$ in all cases). The ketone group in the cascade product was reduced asymmetrically to a chiral secondary hydroxy group (Table 1) [26].

In this case, the role of $\mathrm{Ph}_{3} \mathrm{CCO}_{2} \mathrm{H}$ as additive is to furnish the conjugate base $\mathrm{Ph}_{3} \mathrm{CO}_{2}{ }^{-}$anion which subsequently deproto- 


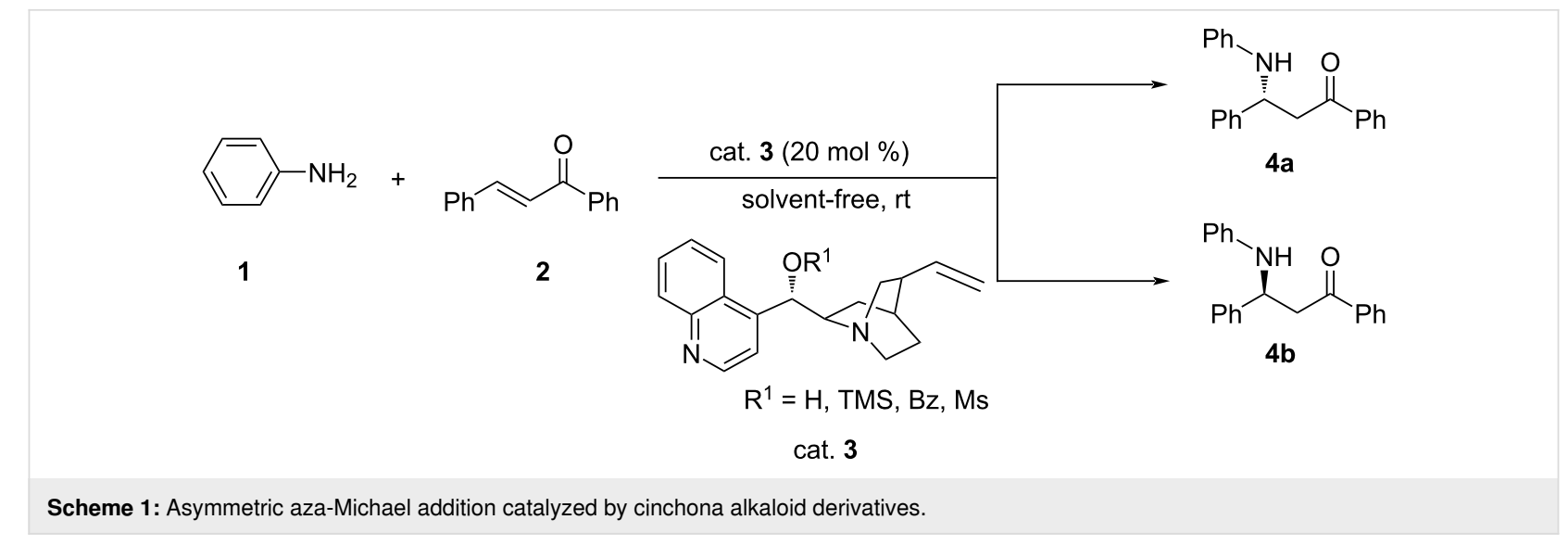

Table 1: Asymmetric cascade aza-Michael-aldol reactions of $\alpha, \beta$-unsaturated ketones with pyrroles.
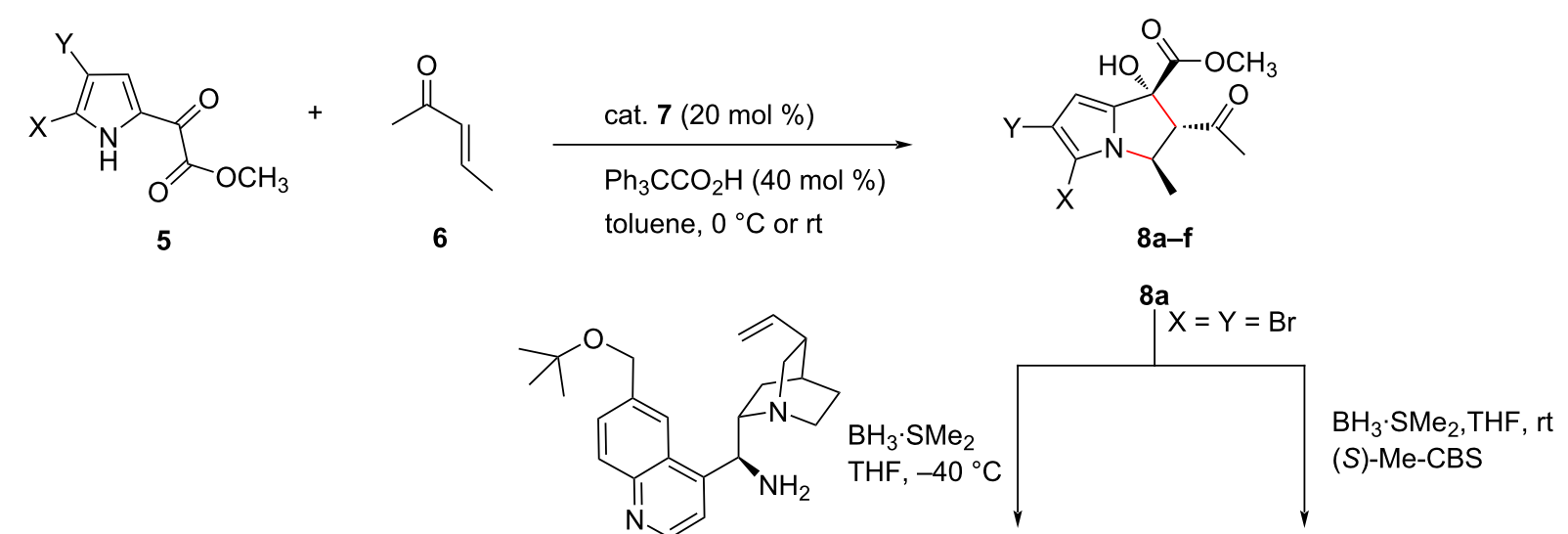

cat. 7

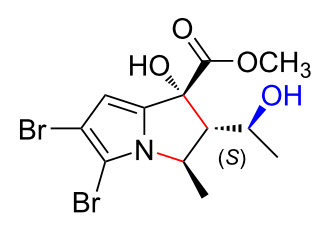

$9 a$

yield $76 \%$; dr 9:1

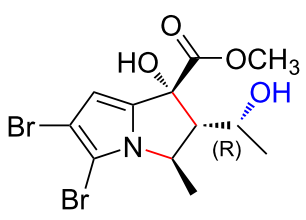

$9 a^{\prime}$

yield $79 \%$; dr 2.9:1

\begin{tabular}{llllll}
\hline 8 & X & $\mathrm{Y}$ & Yield [\%] & ee [\%] & $\mathrm{dr}$ \\
\hline a & $\mathrm{Br}$ & $\mathrm{Br}$ & 86 & 91 & $>20: 1$ \\
b & $\mathrm{Cl}$ & $\mathrm{Br}$ & 91 & 90 & $>20: 1$ \\
c & $\mathrm{I}$ & $\mathrm{Br}$ & 75 & 92 & $>20: 1$ \\
d & $\mathrm{Br}$ & $\mathrm{Cl}$ & 86 & 90 & $>20: 1$ \\
e & $\mathrm{Cl}$ & $\mathrm{I}$ & 73 & 90 & $>20: 1$ \\
$\mathbf{f}$ & $\mathrm{I}$ & $\mathrm{I}$ & 70 & 92 & $>20: 1$
\end{tabular}

nates pyrrole to provide the stronger nucleophilic pyrrolide anion [27].

Similarly, Liu et al. accomplished an asymmetric intramolecular aza-Michael addition of various enone carbamates $\mathbf{1 0}$ using a chiral cinchona-based primary-tertiary diamine as catalyst to obtain 2-substituted piperidines 12 in good yields (75-95\%) with up to $99 \%$ ee. Several sulfonic acids and carboxylic acids were tested as co-catalysts and trifluoroacetic acid (TFA) was found to give the best results [28]. Here the role of the co-catalyst is to assist in the formation of the iminium intermediate (Table 2) [29]. It appears that in this case, both activation mech- 
Table 2: Intramolecular aza-Michael addition of conjugated ketones.<smiles>[R]C(=O)/C=C/CCC([R])([R])CNCC</smiles><smiles>[Mg][Mg]</smiles>

10

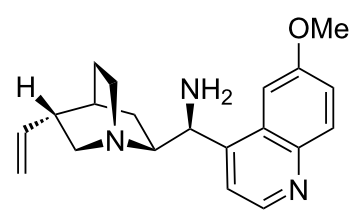

cat. 11<smiles>[R7]C[C@H]1CCC([R1])CN1[R]</smiles>

$12 \mathrm{a}-\mathrm{j}$

\begin{tabular}{llllll}
\hline $\mathbf{1 2}$ & $\mathrm{R}^{1}$ & $\mathrm{R}^{2}$ & $\mathrm{R}^{3}$ & Yield [\%] & ee [\%] \\
\hline $\mathbf{a}$ & $\mathrm{Me}$ & $\mathrm{H}$ & $\mathrm{Cbz}$ & 95 & $98(R)$ \\
$\mathbf{b}$ & $\mathrm{Me}$ & $\mathrm{H}$ & $\mathrm{Boc}$ & 94 & 90 \\
$\mathbf{c}$ & $\mathrm{Me}$ & $\mathrm{Me}$ & $\mathrm{Cbz}$ & 97 & 99 \\
$\mathbf{d}$ & $\mathrm{Et}$ & $\mathrm{H}$ & $\mathrm{Cbz}$ & 94 & $96(R)$ \\
$\mathbf{e}$ & $\mathrm{H}$ & $\mathrm{Cbz}$ & 96 & 99 \\
$\mathbf{f}$ & $\mathrm{H}$ & $\mathrm{H}$ & $\mathrm{Cbz}$ & 93 & 96 \\
$\mathbf{g}$ & $\mathrm{Ph}$ & $\mathrm{H}$ & $\mathrm{Cbz}$ & 95 & 96 \\
$\mathbf{h}$ & $4-\mathrm{Me}-\mathrm{C}_{6} \mathrm{H}_{4}$ & $\mathrm{H}$ & $\mathrm{Cbz}$ & 75 & $\mathrm{ND}$ \\
$\mathbf{i}$ & $4-\mathrm{MeO}-\mathrm{C}_{6} \mathrm{H}_{4}$ & $\mathrm{H}$ & $\mathrm{Cbz}$ & trace & 85 \\
$\mathbf{j}$ & $4-\mathrm{O}_{2} \mathrm{~N}-\mathrm{C}_{6} \mathrm{H}_{4}$ & $\mathrm{H}$ & $\mathrm{Cbz}$ & 80 & \\
\hline
\end{tabular}

aThe starting material was mainly recovered. ${ }^{\mathrm{b}} \mathrm{ND}=$ not determined.

anisms, namely through hydrogen bonding and iminium ion formation are operating.

Using the same chiral cinchona-based primary-tertiary diamine as catalyst (cat. 11), Zhai et al. developed a highly efficient intramolecular enantioselective aza-Michael addition of carbamates, sulfonamides and acetamides 13 bearing an $\alpha, \beta$-unsaturated ketone to synthesize a series of 2 -substituted five- and sixmembered heterocycles in good yields (up to 99\%) and excellent enantioselectivity (92-97.5\% ee) (Table 3). As in an earlier

Table 3: Intramolecular enantioselective aza-Michael addition.

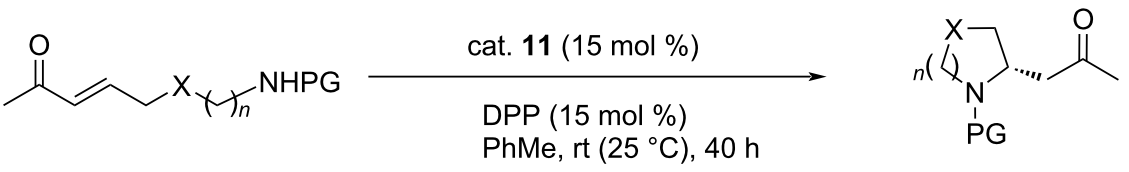

13

$14 a-d$

\begin{tabular}{llllll}
\hline 14 & X & $n$ & PG & Yield [\%] \\
\hline a & O & 2 & Boc [\%] & 97 \\
b & O & 1 & Boc & 96 & 94 \\
c $^{\text {b }}$ & $\mathrm{S}$ & 2 & Cbz & 55 & 95 \\
d & $\mathrm{S}$ & 1 & Cbz & 91 & 92 \\
\hline
\end{tabular}

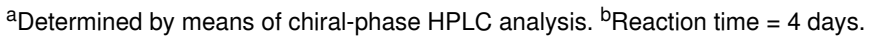


case [29], several acids were tested as co-catalysts and trifluoroacetic acid and diphenyl hydrogenphosphate (DPP) were found to give the best results [30].

Cheng et al. reported an intramolecular 6-exo-trig aza-MR of hydroxylamine-derived enone $\mathbf{1 5}$ for the synthesis of chiral 3-substituted 1,2-oxazinanes $\mathbf{1 6}$. The catalyst $\mathbf{1 1}$ was used in this case also and pentafluoropropionic acid (PFP) was used as a co-catalyst. In the presence of 1,4-dioxane solvent, products chiral 3-substituted 1,2-oxazinanes (16) were obtained in $99 \%$ yield with good ee of $96 \%$ (Scheme 2) [31].
Following a similar strategy, Ma et al. accomplished a highly enantioselective aza-Michael addition of 4-nitrophthalimide (17) with $\alpha, \beta$-unsaturated ketones 18 using 9-epi-9-amino-9deoxyquinine 19 as the catalyst, the corresponding Michael adducts being obtained in moderate to good yields (49-98\%) with excellent ee (95-99\%) (Table 4) [32].

Jakkampudi et al. [33] adopted a different approach for the use of cinchona-based organocatalysts. Instead of using the cinchona derivative alone, they employed a mixture of cinchona derivative and amino acid such as D-proline, termed as the<smiles>CC(=O)C=CCCCONC(=O)OCc1ccccc1</smiles>

Scheme 2: Intramolecular 6-exo-trig aza-Michael addition reaction.

Table 4: Asymmetric aza-Michael addition of 4-nitrophthalimide to $\alpha, \beta$-unsaturated ketones.<smiles>O=C1NC(=O)c2cc([N+](=O)[O-])ccc21</smiles>

17<smiles>[R]C=CC([R])=O</smiles>

18

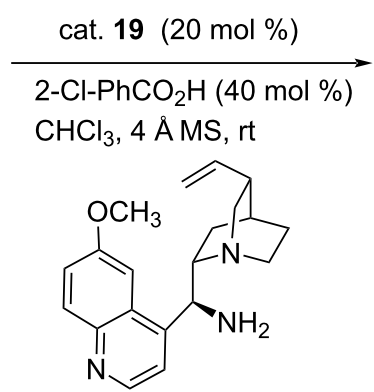

cat. 19<smiles>[R]C(=O)CC([R1])N1C(=O)c2ccc([N+](=O)[O-])cc2C1=O</smiles>

20a-p

\begin{tabular}{lllll}
\hline $\mathbf{2 0}$ & $\mathrm{R}^{1}$ & $\mathrm{R}^{2}$ & Yield [\%] & ee [\%] \\
\hline $\mathbf{a}$ & $\mathrm{Ph}$ & $\mathrm{Ph}$ & 55 & $>99$ \\
$\mathbf{b}$ & $2-\mathrm{Cl}_{-} \mathrm{C}_{6} \mathrm{H}_{4}$ & $\mathrm{Ph}$ & 61 & 95 \\
$\mathbf{c}$ & $3-\mathrm{Cl}_{6} \mathrm{H}_{4}$ & $\mathrm{Ph}$ & 65 & $98(\mathrm{~s})$ \\
$\mathbf{d}$ & $4-\mathrm{Cl}-\mathrm{C}_{6} \mathrm{H}_{4}$ & $\mathrm{Ph}$ & 56 & 99 \\
$\mathbf{e}$ & $4-\mathrm{F}-\mathrm{C}_{6} \mathrm{H}_{4}$ & $\mathrm{Ph}$ & 60 & $>99$ \\
$\mathbf{f}$ & $4-\mathrm{Br}_{6} \mathrm{C}_{4} \mathrm{H}_{4}$ & $\mathrm{Ph}$ & 62 & 99 \\
$\mathbf{g}$ & $4-\mathrm{Me}_{6}-\mathrm{C}_{6} \mathrm{H}_{4}$ & $\mathrm{Ph}$ & 69 & 99 \\
$\mathbf{h}$ & $4-\mathrm{NO}_{2}-\mathrm{C}_{6} \mathrm{H}_{4}$ & $\mathrm{Ph}$ & 49 & $>99$ \\
$\mathbf{i}$ & $\mathrm{Ph}$ & $4-\mathrm{Cl}-\mathrm{C}_{6} \mathrm{H}_{4}$ & 54 & 99 \\
$\mathbf{j}$ & $4-\mathrm{F}-\mathrm{C}_{6} \mathrm{H}_{4}$ & $4-\mathrm{F}-\mathrm{C}_{6} \mathrm{H}_{4}$ & 71 & 99 \\
$\mathbf{k}$ & $\mathrm{Pr}$ & $\mathrm{Me}$ & 75 & 97 \\
$\mathbf{l}$ & $n-\mathrm{Pr}$ & $\mathrm{Me}$ & 88 & 96 \\
$\mathbf{m}$ & $n-\mathrm{Bu}$ & $\mathrm{Me}$ & 89 & 95 \\
$\mathbf{n}$ & $n-\mathrm{Pen}$ & $\mathrm{Me}$ & 98 & 95 \\
$\mathbf{0}$ & $n-\mathrm{Hex}$ & $\mathrm{Me}$ & 90 & 96 \\
$\mathbf{p}$ & $\mathrm{Me}$ & $\mathrm{Et}$ & 51 & 95
\end{tabular}


modularly designed organocatalyst (MDO) for the synthesis of bridged tetrahydroisoquinoline derivatives. It was perceived that the MDO self-assembled in situ from amino acids and cinchona alkaloid derivatives. For example, on reacting $(E)-2-$ [2-(3-aryl-3-oxoprop-1-en-1-yl)phenyl]acetaldehydes 21 with ethyl or benzyl (E)-2-[(4-methoxyphenyl)imino]acetates 22 in the presence of the MDO 23/24 (quinidinethiourea + D-proline), instead of the expected domino Mannich/Michael product, the bridged tetrahydroisoquinoline product 25a was obtained in high yield (90\%) and excellent dr (94:6) and ee value (99\%) (Table 5). The controlled reactions using $\mathbf{2 3}$ and $\mathbf{2 4}$ as the catalyst gave the product in very poor yield. It was concluded that the catalytic activity of the MDO was the result of the cooperative action of both constituents. Several examples of such
MDOs are included in the paper. The reported yield varies from $56-90 \%$ with excellent ee $\approx 99 \%$ in all cases.

\subsection{Reactions catalyzed by chiral squaramide derivatives}

Squaramides are related to cinchona alkaloids but are much more effective organocatalysts than the latter due to the ability of dual hydrogen bonding besides a tertiary nitrogen atom of quinuclidine nucleus which may serve both as an H-bond acceptor and a base in asymmetric Michael addition reactions [34,35].

In 2015, Zhao et al. synthesized spiro[pyrrolidine-3,3'-oxindoles] 29 in single step by asymmetric cascade aza-Michael/

Table 5: Diastereoselective synthesis of bridged 1,2,3,4-tetrahydroisoquinoline derivatives using modularly designed organocatalyst.<smiles>[R]C(=O)/C=C/c1ccc([R])cc1CC=O</smiles>

21<smiles></smiles>

cat. 23/24 toluene, rt, $3 \mathrm{~h}$<smiles>[R]OC(=O)C1c2ccc([R])cc2C(CC([R])=O)C1C(=O)O</smiles>

25a-o<smiles>C=CC1CC2CCN1CC2[C@H](NC(=S)NCCC)c1ccnc2ccc(OC)cc12</smiles><smiles>O=C(O)C1CCCN1</smiles>

cat. 23

cat. 24

\begin{tabular}{|c|c|c|c|c|c|c|}
\hline 25 & $\mathrm{R}^{1}$ & $\mathrm{R}^{2}$ & $\mathrm{R}^{3}$ & Yield [\%] & ee [\%] & $\mathrm{dr}$ \\
\hline $\mathbf{a}$ & $\mathrm{C}_{6} \mathrm{H}_{5}$ & $\mathrm{H}$ & Et & 90 & $>99$ & $94: 6$ \\
\hline b & 4-F-C ${ }_{6} \mathrm{H}_{4}$ & $\mathrm{H}$ & Et & 81 & $>99$ & $97: 3$ \\
\hline c & $4-\mathrm{Cl}-\mathrm{C}_{6} \mathrm{H}_{4}$ & $\mathrm{H}$ & Et & 90 & 99 & $92: 8$ \\
\hline d & $4-\mathrm{Br}-\mathrm{C}_{6} \mathrm{H}_{4}$ & $\mathrm{H}$ & Et & 80 & $>99$ & $90: 10$ \\
\hline e & $4-\mathrm{NC}-\mathrm{C}_{6} \mathrm{H}_{4}$ & $\mathrm{H}$ & Et & 77 & 99 & $87: 13$ \\
\hline$f$ & $4-\mathrm{Me}-\mathrm{C}_{6} \mathrm{H}_{4}$ & $\mathrm{H}$ & Et & 76 & 99 & $97: 3$ \\
\hline g & $4-\mathrm{MeO}-\mathrm{C}_{6} \mathrm{H}_{4}$ & $\mathrm{H}$ & Et & 79 & 97 & $96: 4$ \\
\hline h & $3-\mathrm{Cl}-\mathrm{C}_{6} \mathrm{H}_{4}$ & $\mathrm{H}$ & Et & 72 & $>99$ & $88: 12$ \\
\hline $\mathbf{i}^{\mathrm{a}}$ & $2-\mathrm{F}-\mathrm{C}_{6} \mathrm{H}_{4}$ & $\mathrm{H}$ & Et & - & - & - \\
\hline $\mathbf{j}^{\mathrm{a}}$ & $2-\mathrm{Cl}-\mathrm{C}_{6} \mathrm{H}_{4}$ & $\mathrm{H}$ & Et & - & - & - \\
\hline $\mathbf{k}$ & $\mathrm{C}_{6} \mathrm{H}_{5}$ & $\mathrm{~F}$ & Et & 73 & $>99$ & $90: 10$ \\
\hline I & $\mathrm{C}_{6} \mathrm{H}_{5}$ & $\mathrm{MeO}$ & Et & 74 & 99 & $91: 9$ \\
\hline m & $\mathrm{Me}$ & $\mathrm{H}$ & Et & 56 & 92 & $86: 14$ \\
\hline $\mathbf{n}$ & $\mathrm{C}_{6} \mathrm{H}_{5}$ & $\mathrm{H}$ & $\mathrm{Bn}$ & 75 & 99 & $89: 11$ \\
\hline 0 & $4-\mathrm{Br}-\mathrm{C}_{6} \mathrm{H}_{4}$ & $\mathrm{H}$ & $\mathrm{Bn}$ & 77 & $>99$ & $93: 7$ \\
\hline
\end{tabular}

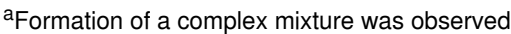


Michael addition reaction between 4-tosylaminobut-2-enoates 27 and 3-ylideneoxindoles 26 catalyzed by a chiral bifunctional tertiary amine, squaramide (cat. 28) which afforded the corresponding adducts in good yields ranging from $72-99 \%$ with excellent diastereoselectivity (up to $>99: 1 \mathrm{dr}$ ) and enantioselectivity (>99\% ee) (Table 6) [36].

In another report, Yang et al. accomplished a highly asymmetric cascade aza-Michael/Michael addition reaction for the synthesis of tetrahydroquinolines and tetrahydrochromanoquinolines catalyzed by a squaramide catalyst. The corresponding adducts were obtained in excellent yields with excel- lent diastereoselectivities and enantioselectivities (up to $>99: 1 \mathrm{dr}, 99 \%$ ee) [37].

Following a similar strategy, Zhou et al. obtained a series of optically active tetrahydrobenzofuro[3,2- $b$ ]quinolines and tetrahydrobenzo[4,5]thieno[3,2- $b]$ quinolines $\mathbf{3 3}$ in high yields ranging from $35-99 \%$ and excellent diastereo- ( $>20: 1 \mathrm{dr}$ ), and enantioselectivities (up to $\approx 99 \%$ ee) (Scheme 3) [38].

Roy et al. accomplished an enantioselective intramolecular azaMichael addition for the synthesis of dihydroisoquinoline and tetrahydropyridines from Michael reaction of ortho-homo-

Table 6: Synthesis of spiro[pyrrolidine-3,3'-oxindoles] via asymmetric cascade aza-Michael reaction catalyzed by squaramide.<smiles>[R]C(=O)C=C1C(=O)N([R6])c2ccc([R])cc21</smiles>

26<smiles>[R]C(=O)/C=C/CN[AsH3]</smiles>

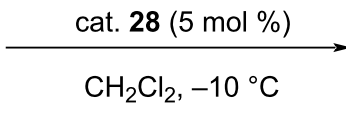

27<smiles>CCC1CN2CCC(C2)[C@H]1[C@H](NC1C(=O)C(=O)C1Nc1cc(C(F)(F)F)cc(C(F)(F)F)c1)c1ccnc2ccc(OC)cc12</smiles><smiles>[R]O[C@H]1N([3H])C[C@@H](CC([R])=O)[C@]12C(=O)N(C(C)(C)C)c1ccc([R])cc12</smiles>

29a-r

cat. 28

\begin{tabular}{|c|c|c|c|c|c|c|}
\hline 29 & $\mathrm{R}^{1}$ & $\mathrm{R}^{2}$ & $\mathrm{R}^{3}$ & Yield [\%] & ee [\%] & $\mathrm{dr}$ \\
\hline a & $\mathrm{Ph}$ & $\mathrm{H}$ & $\mathrm{Me}$ & 72 & 98 & $96: 4$ \\
\hline b & $\mathrm{Ph}$ & $\mathrm{H}$ & $\mathrm{O} t-\mathrm{Bu}$ & 99 & $>99$ & $93: 7$ \\
\hline C & $\mathrm{Ph}$ & $\mathrm{H}$ & OBn & 99 & $>99$ & $88: 12$ \\
\hline d & $\mathrm{Ph}$ & $\mathrm{H}$ & OEt & 99 & $>99$ & $65: 35$ \\
\hline e & $4-\mathrm{FC}_{6} \mathrm{H}_{4}$ & $\mathrm{H}$ & $\mathrm{O} t-\mathrm{Bu}$ & 99 & $>99$ & $88: 12$ \\
\hline f & $4-\mathrm{ClC}_{6} \mathrm{H}_{4}$ & $\mathrm{H}$ & $\mathrm{O} t-\mathrm{Bu}$ & 99 & $>99$ & $89: 11$ \\
\hline g & $2-\mathrm{BrC}_{6} \mathrm{H}_{4}$ & $\mathrm{H}$ & $\mathrm{O} t-\mathrm{Bu}$ & 91 & $>99$ & $85: 15$ \\
\hline $\mathbf{h}$ & $4-\mathrm{BrC}_{6} \mathrm{H}_{4}$ & $\mathrm{H}$ & $\mathrm{O} t-\mathrm{Bu}$ & 99 & $>99$ & $92: 8$ \\
\hline i & 4- $\mathrm{MeC}_{6} \mathrm{H}_{4}$ & $\mathrm{H}$ & $\mathrm{O} t-\mathrm{Bu}$ & 95 & $>99$ & $96: 4$ \\
\hline j & 3- $\mathrm{MeOC}_{6} \mathrm{H}_{4}$ & $\mathrm{H}$ & $\mathrm{O} t-\mathrm{Bu}$ & 88 & $>99$ & $92: 8$ \\
\hline $\mathbf{k}$ & 4- $\mathrm{MeOC}_{6} \mathrm{H}_{4}$ & $\mathrm{H}$ & $\mathrm{O} t-\mathrm{Bu}$ & 94 & $>99$ & $89: 11$ \\
\hline I & 2-naphthyl & $\mathrm{H}$ & $\mathrm{O} t-\mathrm{Bu}$ & 92 & $>99$ & $89: 11$ \\
\hline $\mathrm{m}$ & 2-thienyl & $\mathrm{H}$ & $\mathrm{O} t-\mathrm{Bu}$ & 96 & $>99$ & $92: 8$ \\
\hline $\mathbf{n}$ & $\mathrm{C}_{6} \mathrm{H}_{5}$ & $\mathrm{~F}$ & $\mathrm{O} t-\mathrm{Bu}$ & 99 & $>99$ & $89: 11$ \\
\hline 0 & $\mathrm{C}_{6} \mathrm{H}_{5}$ & $\mathrm{Cl}$ & $\mathrm{O} t-\mathrm{Bu}$ & 99 & $>99$ & $88: 12$ \\
\hline $\mathbf{p}$ & $\mathrm{C}_{6} \mathrm{H}_{5}$ & $\mathrm{Br}$ & $\mathrm{O} t-\mathrm{Bu}$ & 99 & $>99$ & $93: 7$ \\
\hline $\mathbf{q}$ & $\mathrm{C}_{6} \mathrm{H}_{5}$ & $\mathrm{Me}$ & $\mathrm{O} t-\mathrm{Bu}$ & 99 & $>99$ & $97: 3$ \\
\hline$r$ & $\mathrm{C}_{6} \mathrm{H}_{5}$ & $\mathrm{OMe}$ & $\mathrm{O} t-\mathrm{Bu}$ & 99 & $>99$ & $99: 1$ \\
\hline
\end{tabular}


<smiles></smiles><smiles>[R]c1ccc(N)c(/C=C/C(=O)P)c1</smiles>
31 $\mathrm{R}^{1}=\mathrm{H}, \mathrm{Me}, \mathrm{OMe}, \mathrm{Br}, \mathrm{Cl}$ $X=O, S$ $\mathrm{R}^{2}=\mathrm{H}, \mathrm{Me}, \mathrm{Br}, \mathrm{Cl}$, cat. $32(20 \mathrm{~mol} \%)$<smiles>[TeH4]</smiles><smiles>O=c1c(N[Al])c(NC2CCCCC2C2CCCCC2)c1=O</smiles>

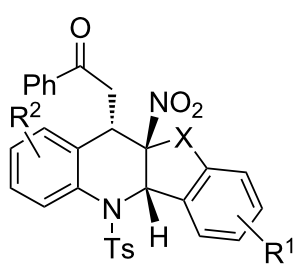

33

yield 35 up to $99 \%$

$\mathrm{dr}>20: 1$

$99 \%$ ee

Scheme 3: Asymmetric aza-Michael/Michael addition cascade reaction of 2-nitrobenzofurans and 2-nitrobenzothiophenes with 2-aminochalcones catalyzed by squaramide derivative.

formyl chalcone with various amines by using squaramide catalyst. The reaction occurred with good yields and excellent enantioselectivity [39].

Similarly, Li et al. reported an asymmetric cascade aza-Michael addition of 2-tosylaminoenones with unsaturated pyrazolones using squaramide as catalyst. The reaction proceeded smoothly under mild conditions to afford the corresponding spiro[pyrazolone-tetrahydroquinolines] in high yields (up to 99\%) with excellent diastereoselectivities (up to $>25: 1 \mathrm{dr}$ ) and high enantioselectivities (up to 65-91\%) [40].
Rajasekar et al. developed an efficient one-pot tandem rhodium(II)/chiral squaramide relay catalysis for the enantioselective construction of dihydro- $\beta$-carbolines $\mathbf{3 7}$ from the Michael reaction of suitably substituted indole derivatives 34 with $N$-sulfonyl-1,2,3-triazoles 35 in good yields (up to $\approx 72 \%$ ) and excellent enantioselectivity (up to $99 \%$ ee) (Table 7) [41].

In an interesting study, Wu et al. screened a number of cinchona derivatives and squaramides for their relative catalytic efficacies for the enantioselective aza-Michael additions between

Table 7: Asymmetric aza-Michael synthesis of dihydro- $\beta$-carbolines.<smiles>Cn1c(/C=C/C(=O)c2ccccc2)cc2ccccc21</smiles>

34

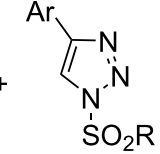

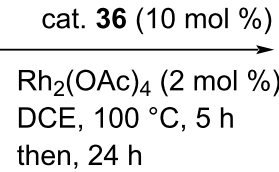

35<smiles>[R]O[N+]1([O-])C=C([Al])c2c(n(C)c3ccccc23)[C@H]1CC(=O)c1ccccc1</smiles>

$37 a, b$<smiles>C=CC1C2CCN1C(c1ccnc3ccccc13)C2Nc1c(NCc2ccccc2)c(=O)c1=O</smiles>

cat. 36

\begin{tabular}{lllll}
\hline 37 & $\mathrm{Ar}$ & $\mathrm{R}$ & Yield [\%] & ee [\%] \\
\hline a & $\mathrm{Ph}$ & $\mathrm{Ph}$ & 71 & 99 \\
b & $\mathrm{Ph}$ & $\mathrm{Me}$ & 72 & 80
\end{tabular}


halogenated 2-hydroxypyridines (pyridin-2(1H)-ones) 38 and $\alpha, \beta$-unsaturated 1,4-diketones or 1,4-ketoesters 39 in different solvents. The best results (yield $96 \%$, ee $>91 \%$ ) were obtained on using squaramide catalyst in chloroform. However, for others, the yields ranged from $50-98 \%$ with good to excellent enantioselectivity (47-98\% ee). The observed results were rationalized with density functional theory calculations (Table 8) [42].

Table 8: Asymmetric aza-Michael synthesis of $N$-substituted 2-pyridones.<smiles>[X]c1ccc(O)nc1</smiles>

38<smiles>[R]C(=O)C=CC([R])=O</smiles>

39

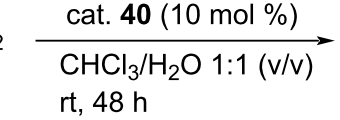<smiles>C=CC1CC2CCN1CC2NC(Nc1c(N[Al])c(=O)c1=O)c1ccnc2ccccc12</smiles><smiles>[R]C(=O)CC(C([R])=O)n1cc([X])ccc1=O</smiles>

$41 a-z$

$$
\mathrm{Ar}=3,5-\left(\mathrm{CF}_{3}\right)_{2} \mathrm{C}_{6} \mathrm{H}_{3}
$$

cat. 40

\begin{tabular}{|c|c|c|c|c|c|}
\hline 41 & $x$ & $\mathrm{R}^{1}$ & $\mathrm{R}^{2}$ & Yield [\%] & ee [\%] \\
\hline a & $5-\mathrm{Br}$ & $\mathrm{Ph}$ & $\mathrm{Ph}$ & 98 & 98 \\
\hline b & I-5 & $\mathrm{Ph}$ & $\mathrm{Ph}$ & 78 & 91 \\
\hline c & $5-\mathrm{F}$ & $\mathrm{Ph}$ & $\mathrm{Ph}$ & 60 & 74 \\
\hline d & $\mathrm{H}$ & $\mathrm{Ph}$ & $\mathrm{Ph}$ & 50 & 44 \\
\hline e & $3-\mathrm{Cl}$ & $\mathrm{Ph}$ & $\mathrm{Ph}$ & 88 & 93 \\
\hline$f$ & $3-\mathrm{Br}$ & $\mathrm{Ph}$ & $\mathrm{Ph}$ & 93 & 92 \\
\hline g & $3-1$ & $\mathrm{Ph}$ & $\mathrm{Ph}$ & 82 & 73 \\
\hline h & $4-\mathrm{Br}$ & $\mathrm{Ph}$ & $\mathrm{Ph}$ & 60 & 47 \\
\hline $\mathbf{i}$ & $6-\mathrm{Cl}$ & $\mathrm{Ph}$ & $\mathrm{Ph}$ & 0 & - \\
\hline j & $5-\mathrm{Cl}$ & $p-\mathrm{F}-\mathrm{C}_{6} \mathrm{H}_{4}$ & $p-\mathrm{F}-\mathrm{C}_{6} \mathrm{H}_{4}$ & 93 & 93 \\
\hline $\mathbf{k}$ & $5-\mathrm{Br}$ & $p-\mathrm{F}-\mathrm{C}_{6} \mathrm{H}_{4}$ & $p-\mathrm{F}-\mathrm{C}_{6} \mathrm{H}_{4}$ & 95 & $>99$ \\
\hline I & I-I & $p-\mathrm{F}-\mathrm{C}_{6} \mathrm{H}_{4}$ & $p-\mathrm{F}-\mathrm{C}_{6} \mathrm{H}_{4}$ & 87 & 97 \\
\hline $\mathbf{m}$ & $3-\mathrm{Cl}$ & $p-\mathrm{F}-\mathrm{C}_{6} \mathrm{H}_{4}$ & $p-\mathrm{F}-\mathrm{C}_{6} \mathrm{H}_{4}$ & 82 & 99 \\
\hline $\mathbf{n}$ & $3-\mathrm{Br}$ & $p-\mathrm{F}-\mathrm{C}_{6} \mathrm{H}_{4}$ & $p-\mathrm{F}-\mathrm{C}_{6} \mathrm{H}_{4}$ & 88 & 97 \\
\hline 0 & $3-1$ & $p-\mathrm{F}-\mathrm{C}_{6} \mathrm{H}_{4}$ & $p-\mathrm{F}-\mathrm{C}_{6} \mathrm{H}_{4}$ & 90 & 99 \\
\hline p & $5-\mathrm{Cl}$ & $p-\mathrm{NC}-\mathrm{C}_{6} \mathrm{H}_{4}$ & $p-\mathrm{NC}-\mathrm{C}_{6} \mathrm{H}_{4}$ & 85 & 94 \\
\hline$q$ & $5-\mathrm{Br}$ & $p-\mathrm{NC}-\mathrm{C}_{6} \mathrm{H}_{4}$ & $p-\mathrm{NC}-\mathrm{C}_{6} \mathrm{H}_{4}$ & 73 & 97 \\
\hline$r$ & $5-\mathrm{Cl}$ & $p-\mathrm{Me}^{-\mathrm{C}_{6}} \mathrm{H}_{4}$ & $p-\mathrm{Me}^{-\mathrm{C}_{6}} \mathrm{H}_{4}$ & 70 & 35 \\
\hline $\mathbf{s}$ & $5-\mathrm{Br}$ & $p-\mathrm{Me}^{-} \mathrm{C}_{6} \mathrm{H}_{4}$ & $p-\mathrm{Me}-\mathrm{C}_{6} \mathrm{H}_{4}$ & 76 & 82 \\
\hline$t$ & I-5 & $p-\mathrm{Me}-\mathrm{C}_{6} \mathrm{H}_{4}$ & $p-\mathrm{Me}-\mathrm{C}_{6} \mathrm{H}_{4}$ & 75 & 67 \\
\hline $\mathbf{u}$ & $5-\mathrm{Cl}$ & $p-\mathrm{MeO}-\mathrm{C}_{6} \mathrm{H}_{4}$ & $p-\mathrm{MeO}-\mathrm{C}_{6} \mathrm{H}_{4}$ & 0 & - \\
\hline $\mathbf{v}$ & $5-\mathrm{Cl}$ & OEt & $\mathrm{Ph}$ & 90 & 78 \\
\hline $\mathbf{w}$ & $5-\mathrm{Br}$ & OEt & $\mathrm{Ph}$ & 82 & 80 \\
\hline $\mathbf{x}$ & $5-\mathrm{F}$ & OEt & $\mathrm{Ph}$ & 83 & 63 \\
\hline$y$ & $3-\mathrm{Cl}$ & OEt & $\mathrm{Ph}$ & 70 & 90 \\
\hline z & $3-\mathrm{Br}$ & OEt & $\mathrm{Ph}$ & 78 & 90 \\
\hline aa & $4-\mathrm{Br}$ & OEt & $\mathrm{Ph}$ & 73 & 60 \\
\hline$a b$ & $3-\mathrm{Cl}$ & OEt & $p-\mathrm{F}-\mathrm{C}_{6} \mathrm{H}_{4}$ & 90 & 80 \\
\hline
\end{tabular}




\subsection{Reactions catalyzed by chiral amines}

$\mathrm{He}$ and co-workers developed heterogeneous synergistic catalysis using chiral amines SBA-15 (cat. 44), which promote azaMichael-Henry cascade reactions between 2-aminobenzaldehydes $\mathbf{4 2}$ and $\beta$-nitrostyrenes $\mathbf{4 3}$ to obtain chiral 3-nitro-1,2dihydroquinolines 45 in good yields with up to $98 \%$ ee (Table 9) [43]

\subsection{Reactions catalyzed by chiral phase-transfer catalysts}

Chiral phase-transfer catalysts (PTC) have been recognized as versatile catalysts for the asymmetric aza-Michael addition reactions. Mahe et al. reported an effective, eco-friendly and cost-effective enantioselective synthesis of 3,5-diarylpyrazolines 49 by using phase-transfer methodology. They carried out a set of reactions between chalcones $\mathbf{4 6}$ and $\mathrm{N}$-tert-butoxycarbonylhydrazine (47) in the presence of cesium carbonate and an $N$-benzylquininium salt as catalyst (cat. 48) (solid-liquid phase-transfer conditions) to give the corresponding adducts in $40-90 \%$ yields with excellent ee of up to $99 \%$ (Table 10 ) [44].

A different type of asymmetric aza-Michael addition was developed by Wang et al. They carried out asymmetric conjugate amination of tert-butylbenzyloxycarbamate (50) to $\beta$-nitro- styrene 51 under neutral phase-transfer conditions in the presence of chiral bifunctional tetraalkylammonium bromide (cat. 52) in water-rich biphasic solvent. The reaction proceeded with high ee values of up to $95 \%$ and very good yields $(\approx 99 \%)$ in all cases (Table 11) [45].

Guo et al. synthesized a variety of benzoindolizidines (56) from $\alpha, \beta$-unsaturated aminoketones 54 through intramolecular domino aza-Michael addition/alkylation reactions. The reactions were carried out in the presence of cinchona alkaloid-derived quaternary ammonium salts (cat. 55) as the phase-transfer catalyst. The products were obtained in high yields (53-93\%) with high enantioselectivities ( $40-76 \%$ ee) (Table 12) [46].

Lebrun et al. developed a new method to synthesize optically active isoindolinones via asymmetric intramolecular aza-MR by using phase-transfer catalysts. Alkenylated benzamide was used as the substrate in this reaction. The resulting compounds were found to be useful intermediates for the synthesis and development of benzodiazepine-receptor agonists [47].

In 2018, Sallio et al. worked on the same reaction by using different PTCs in order to improve yield and diastereomeric excess. They incorporated PTC and chiral auxiliary and reacted

Table 9: Asymmetric aza-Michael-Henry cascade reaction.<smiles>[R1]c1ccc(C=O)cc1N</smiles>

42<smiles>[R]C=C[N+](=O)[O-]</smiles>

43<smiles>[TeH4]</smiles><smiles>CCN1CCCC1CNCCC[Si]1C2C(O)C1C(C(O)C(C)O)C2C(C)O</smiles>

SBA-15-AEP

cat. 44

\begin{tabular}{lllll}
\hline $\mathbf{4 5}$ & $\mathrm{R}^{1}$ in $\mathbf{4 2}$ & $\mathrm{R}^{2}$ & Yield [\%] $^{\mathrm{a}}$ & ee [\%] \\
\hline $\mathbf{a}$ & $\mathrm{H}$ & $2,3-(\mathrm{MeO})_{2}-\mathrm{C}_{6} \mathrm{H}_{3}$ & $67(65)$ & $98(98)$ \\
$\mathbf{b}$ & $\mathrm{H}$ & $4-\mathrm{Me}-\mathrm{C}_{6} \mathrm{H}_{4}$ & $60(59)$ & $90(93)$ \\
c & $\mathrm{H}$ & $2,4-(\mathrm{Cl})_{2}-\mathrm{C}_{6} \mathrm{H}_{3}$ & $45(40)$ & $97(96)$ \\
d & $\mathrm{H}$ & $3,4-(\mathrm{Cl})_{2}-\mathrm{C}_{6} \mathrm{H}_{3}$ & $42(38)$ & $95(97)$ \\
$\mathbf{e}$ & $3-\mathrm{MeO}$ & $\mathrm{Ph}$ & $52(50)$ & $95(99)$ \\
$\mathbf{f}$ & $5-\mathrm{Cl}$ & $\mathrm{Ph}$ & $55(53)$ & $98(99)$ \\
$\mathbf{g}$ & $3,5-\mathrm{Br}_{2}$ & $\mathrm{Ph}$ & $68(67)$ & $99(98)$ \\
\hline
\end{tabular}

aDetermined by ${ }^{1} \mathrm{H}$ NMR. ${ }^{b}$ Determined by HPLC. The data in parentheses are reproduced results. 
Table 10: Asymmetric aza-Michael addition for the formation of (S)-(-)-pyrazoline.

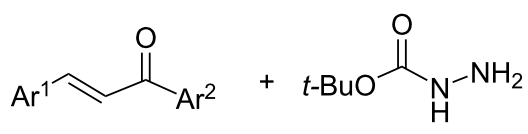

46

47

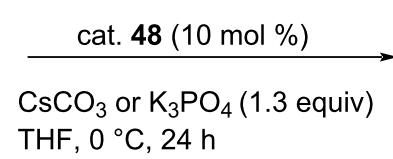
$\mathrm{THF}, 0^{\circ} \mathrm{C}, 24 \mathrm{~h}$

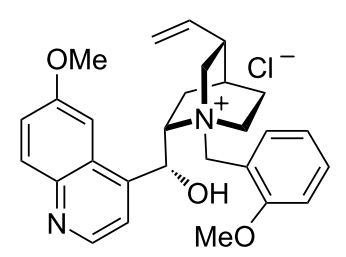

$t$-BuO<smiles>CC(=O)N1N=C([Al])CC1[18O]</smiles>

49a-n

cat. 48

\begin{tabular}{lllll}
\hline $\mathbf{4 9}$ & $\mathrm{Ar}^{1}$ & $\mathrm{Ar}^{2}$ & Yield [\%] & ee [\%] \\
\hline $\mathbf{a}$ & $\mathrm{Ph}$ & $\mathrm{Ph}$ & 77 & $92(-)$ \\
$\mathbf{b}$ & $\mathrm{Ph}$ & $4-\mathrm{MeOC}_{6} \mathrm{H}_{4}$ & 71 & $90(-)$ \\
$\mathbf{c}$ & $\mathrm{Ph}$ & $4-\mathrm{FC}_{6} \mathrm{H}_{4}$ & 72 & $90(-)$ \\
$\mathbf{d}$ & $\mathrm{Ph}$ & $4-\mathrm{FC}_{6} \mathrm{H}_{4}$ & 62 & $92(-)$ \\
$\mathbf{e}$ & $\mathrm{Ph}$ & $2-\mathrm{MeOC}_{6} \mathrm{H}_{4}$ & 89 & $92(-)$ \\
$\mathbf{f}$ & $\mathrm{Ph}$ & $2-\mathrm{MeOC}_{6} \mathrm{H}_{4}$ & 52 & $94(-)$ \\
$\mathbf{g}$ & $\mathrm{Ph}$ & 2 -thienyl & 66 & $87(-)$ \\
$\mathbf{h}$ & $\mathrm{Ph}$ & $2-$ thienyl & 60 & $91(-)$ \\
$\mathbf{i}$ & $\mathrm{Ph}$ & $3,4-(\mathrm{Cl})_{2} \mathrm{C}_{6} \mathrm{H}_{3}$ & $40(62)^{\mathrm{a}}$ & $92(-)$ \\
$\mathbf{j}$ & $4-\mathrm{MeOC}_{6} \mathrm{H}_{4}$ & $\mathrm{Ph}$ & 60 & $89(+)$ \\
$\mathbf{k}$ & $4-\mathrm{ClC}_{6} \mathrm{H}_{4}$ & $\mathrm{Ph}$ & 70 & $88(-)$ \\
$\mathbf{I}$ & $2-\mathrm{MeC}_{6} \mathrm{H}_{4}$ & $\mathrm{Ph}$ & 62 & $89(-)$ \\
$\mathbf{m}$ & $3-\mathrm{MeOC}_{6} \mathrm{H}_{4}$ & $\mathrm{Ph}$ & 61 & $91(-)$ \\
$\mathbf{n}$ & $2-$ thienyl & $\mathrm{Ph}$ & 46 & $78(-)$ \\
\hline
\end{tabular}

aYield determined by NMR analysis of the crude reaction mixture using an internal standard.

Table 11: Asymmetric aza-Michael addition reaction catalyzed by phase-transfer catalyst.

$$
\text { Boc } \text { N }^{-O B n}+
$$

50<smiles>[R]C=C[N+](=O)[O-]</smiles>

51
$\underset{\mathrm{H}_{2} \mathrm{O} / \text { toluene } 10: 1}{\stackrel{(\mathrm{S}) \text { cat. } 52(0.05-1 \mathrm{~mol} \%)}{\longrightarrow}}$

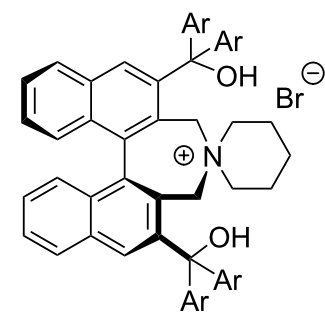<smiles>[R]C(C[N+](=O)[O-])N(OCc1ccccc1)C(=O)OCc1ccccc1</smiles>

$53 a-j$

$$
\mathrm{Ar}=3,5-\left[3,5-\left(\mathrm{CF}_{3}\right)_{2} \mathrm{C}_{6} \mathrm{H}_{3}\right]_{2} \mathrm{C}_{6} \mathrm{H}_{3}
$$

cat. 52

\begin{tabular}{llllllll}
\hline 53 & Conditions $^{\mathrm{a}}$ & & $\mathrm{R}$ & Yield (\%) & & ee [\%] \\
\hline & A & B & & A & B & A \\
\hline a & A & B & Ph & B & 91 & 90 & 90
\end{tabular}


Table 11: Asymmetric aza-Michael addition reaction catalyzed by phase-transfer catalyst. (continued)

\begin{tabular}{|c|c|c|c|c|c|c|c|}
\hline b & $A$ & $\mathrm{~B}$ & 4-Me- $\mathrm{C}_{6} \mathrm{H}_{4}$ & 93 & 90 & 90 & 91 \\
\hline C & A & $\mathrm{B}$ & $4-\mathrm{BrC}_{6} \mathrm{H}_{4}$ & 91 & 89 & 91 & 94 \\
\hline d & $A$ & $\mathrm{~B}$ & $3-\mathrm{FC}_{6} \mathrm{H}_{4}$ & 70 & 62 & 90 & 93 \\
\hline e & A & $\mathrm{B}$ & 4-TBSOC $6{ }_{6} \mathrm{H}_{4}$ & 94 & 92 & 92 & 95 \\
\hline$f$ & $A$ & B & 2-naphthyl & 85 & 70 & 90 & 91 \\
\hline g & $A$ & $\mathrm{~B}$ & 2-thienyl & 93 & 81 & 90 & 94 \\
\hline $\mathbf{h}$ & A & B & 2-furyl & 90 & 82 & 90 & 93 \\
\hline i & $A$ & $\mathrm{~B}$ & $\left(\mathrm{CH}_{3}\right)_{2} \mathrm{CHCH}_{2}$ & 95 & 99 & 77 & 82 \\
\hline j & $A$ & B & $t-\mathrm{Bu}$ & 97 & 99 & 79 & 83 \\
\hline
\end{tabular}

aConditions A: cat. $(0.05 \mathrm{~mol} \%)$ at $\mathrm{rt}$ or $0{ }^{\circ} \mathrm{C}$, conditions B: cat. $(1 \mathrm{~mol} \%)$ at $0{ }^{\circ} \mathrm{C}$.

Table 12: Asymmetric aza-Michael/alkylation reaction catalyzed by cinchona alkaloid-derived quaternary ammonium salts.<smiles>[R]C(=O)/C=C/C1=C(CCNC(=O)CCl)C=C[R1]C=C1</smiles>

54

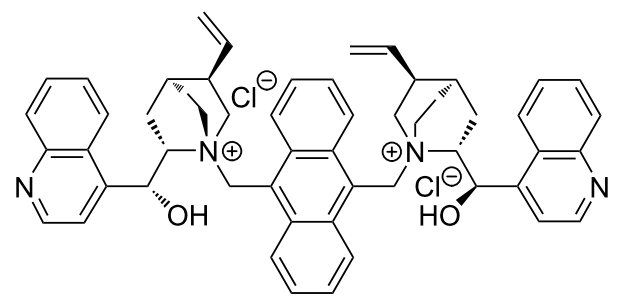

$56 a-0$

cat. 55

\begin{tabular}{|c|c|c|c|c|}
\hline 56 & $\mathrm{R}^{1}$ & $\mathrm{R}^{2}$ & Yield [\%] & ee [\%] \\
\hline $\mathbf{a}$ & $\mathrm{H}$ & $\mathrm{Ph}$ & 91 & 76 \\
\hline b & $\mathrm{H}$ & $4-\mathrm{MeC}_{6} \mathrm{H}_{4}$ & 84 & 69 \\
\hline c & $\mathrm{H}$ & 4- $\mathrm{MeOC}_{6} \mathrm{H}_{4}$ & 53 & 65 \\
\hline d & $\mathrm{H}$ & $\mathrm{Ph}-\mathrm{C}_{6} \mathrm{H}_{4}$ & 71 & 75 \\
\hline e & $\mathrm{H}$ & 2-naphthyl & 91 & 72 \\
\hline f & $\mathrm{H}$ & $4-\mathrm{FC}_{6} \mathrm{H}_{4}$ & 88 & 50 \\
\hline g & $\mathrm{H}$ & $4-\mathrm{ClC}_{6} \mathrm{H}_{4}$ & 93 & 80 \\
\hline h & $\mathrm{H}$ & $4-\mathrm{BrC}_{6} \mathrm{H}_{4}$ & 93 & 65 \\
\hline i & $\mathrm{H}$ & 2-furyl & 87 & 54 \\
\hline j & $\mathrm{H}$ & 2-thienyl & 91 & 68 \\
\hline $\mathbf{k}$ & $\mathrm{H}$ & $2-P y$ & 89 & 63 \\
\hline I & $2-\mathrm{Br}$ & $\mathrm{Ph}$ & 86 & 85 \\
\hline $\mathbf{m}$ & $2-\mathrm{NO}_{2}$ & $\mathrm{Ph}$ & 75 & 85 \\
\hline $\mathbf{n}$ & 3-MeO & $\mathrm{Ph}$ & 87 & 40 \\
\hline 0 & $\mathrm{H}$ & $\mathrm{Me}$ & - & - \\
\hline
\end{tabular}

a variety of chiral phthalimidines $\mathbf{5 7}$ to obtain isoindolinones $\mathbf{5 9}$ in good yields $(\approx 85 \%)$ with excellent de ranging $48-96 \%$ (Table 13) [48].
1.5 Catalysis by chiral bifunctional thioureas

Thioureas constitute one of the most important class of organocatalysts [49]. 
Table 13: Asymmetric aza-Michael synthesis of isoindolinones.

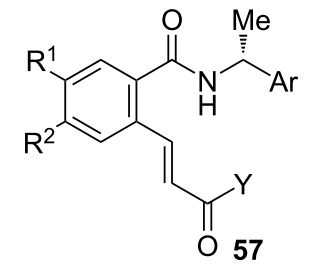

$$
\begin{aligned}
& \underset{\text { cat. } 58(10 \mathrm{~mol} \%)}{\stackrel{\mathrm{Cs}_{2} \mathrm{CO}_{3}(1.3 \text { equiv })}{\text { toluene, } \mathrm{rt}, 36 \mathrm{~h}}}
\end{aligned}
$$

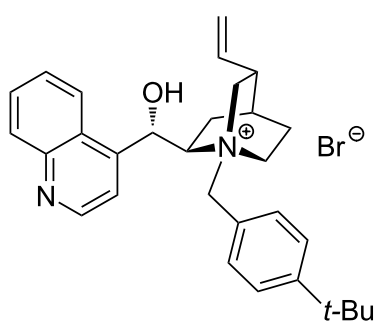

cat. 58

\begin{tabular}{|c|c|c|c|c|c|c|}
\hline 59 & $\mathrm{R}^{1}$ & $\mathrm{R}^{2}$ & $\mathrm{Ar}$ & $Y$ & Yield [\%] & de [\%] \\
\hline $\mathbf{a}$ & $\mathrm{H}$ & $\mathrm{H}$ & $\mathrm{Ph}$ & & 75 & $>96$ \\
\hline b & $\mathrm{H}$ & $\mathrm{H}$ & $\mathrm{Ph}$ & & 78 & $>96$ \\
\hline c & $\mathrm{H}$ & $\mathrm{H}$ & $\mathrm{Ph}$ & & 79 & 56 \\
\hline d & $\mathrm{H}$ & $\mathrm{H}$ & $\mathrm{Ph}$ & & 80 & 44 \\
\hline e & $\mathrm{H}$ & $\mathrm{H}$ & PMPa & & 82 & 82 \\
\hline f & $\mathrm{H}$ & $\mathrm{H}$ & PMP & & 80 & 98 \\
\hline g & $\mathrm{H}$ & $\mathrm{H}$ & PMP & & 85 & 98 \\
\hline h & $\mathrm{H}$ & $\mathrm{H}$ & PMP & & 83 & 48 \\
\hline $\mathbf{i}$ & $\mathrm{H}$ & $\mathrm{H}$ & PMP & & 79 & 67 \\
\hline j & $\mathrm{MeO}$ & $\mathrm{MeO}$ & PMP & & 78 & 98 \\
\hline
\end{tabular}<smiles></smiles>

aPMP $=p$-methoxyphenyl.

Wang et al. reported a cascade aza-Michael/Michael reaction of anilines $\mathbf{6 0}$ to nitroolefin enoates $\mathbf{6 1}$ using chiral bifunctional thiourea as catalyst (cat. 62). It provided a mild and efficient approach to the synthesis of three stereocentered polysubstituted chiral 4-aminobenzopyrans 63 in high yields (71-96\%) with excellent stereoselectivities of up to $>99 \%$ ee (Table 14) [50].
In an interesting report, five organocatalysts belonging to three categories, namely cinchona alkaloid bases, bifunctional squaramides and thioureas were screened for the enantioselective $N$-alkylation of isoxazolin-5-ones via a 1,6-aza-Michael addition of isoxazolin-5-ones 64 to $p$-quinone methides ( $p$-QMs) $\mathbf{6 5}$ to give isoxazolin-5-ones $\mathbf{6 7}$ bearing a chiral diarylmethyl 
Table 14: Asymmetric aza-Michael addition reaction catalysed by chiral bifunctional thiourea.

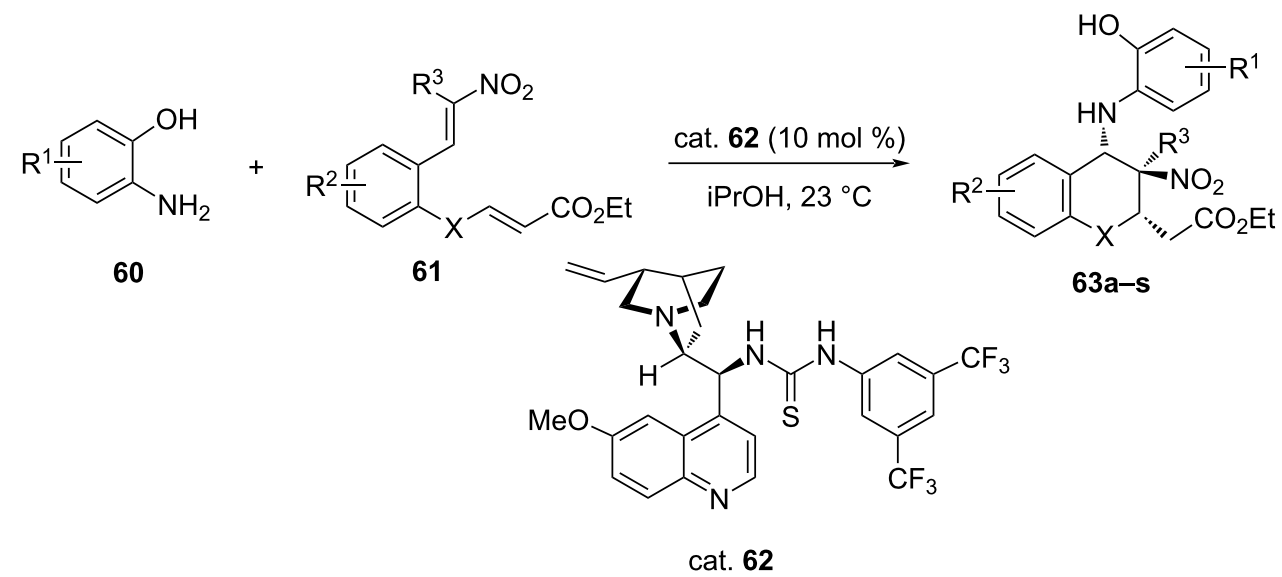

cat. 62

\begin{tabular}{|c|c|c|c|c|c|c|c|}
\hline 63 & $\mathrm{R}^{1}$ & $\mathrm{R}^{2}$ & $\mathrm{R}^{3}$ & $x$ & Yield [\%] & ee [\%] & $d r$ \\
\hline a & $\mathrm{H}$ & $\mathrm{H}$ & $\mathrm{Me}$ & O & 96 & 96 & $>95: 5$ \\
\hline b & $\mathrm{H}$ & $4-\mathrm{F}$ & $\mathrm{Me}$ & 0 & 71 & $>99$ & $>95: 5$ \\
\hline c & $\mathrm{H}$ & $4-\mathrm{Cl}$ & $\mathrm{Me}$ & 0 & 92 & 94 & $>95: 5$ \\
\hline d & $\mathrm{H}$ & $4-\mathrm{Br}$ & $\mathrm{Me}$ & 0 & 84 & 94 & $>95: 5$ \\
\hline e & $\mathrm{H}$ & 4-Me & $\mathrm{Me}$ & O & 92 & 94 & $>95: 5$ \\
\hline f & $\mathrm{H}$ & 4-MeO & $\mathrm{Me}$ & 0 & 94 & 94 & $95: 5$ \\
\hline g & 4-Me & $5-\mathrm{MeO}$ & $\mathrm{Me}$ & 0 & 83 & 96 & $>95: 5$ \\
\hline h & 4-Me & $4-\mathrm{Br}$ & $\mathrm{Me}$ & $\mathrm{O}$ & 82 & 94 & $>95: 5$ \\
\hline i & 5-Me & $4-\mathrm{Br}$ & $\mathrm{Me}$ & 0 & 85 & 93 & $>95: 5$ \\
\hline j & 6-Me & $\mathrm{H}$ & $\mathrm{Me}$ & 0 & 94 & 93 & $>95: 5$ \\
\hline k & 6-Me & $5-\mathrm{MeO}$ & $\mathrm{Me}$ & 0 & 81 & 94 & $>95: 5$ \\
\hline I & 4-Me & $\mathrm{H}$ & $\mathrm{Me}$ & 0 & 94 & 96 & $>95: 5$ \\
\hline m & $4-\mathrm{Br}$ & $\mathrm{H}$ & $\mathrm{Me}$ & 0 & 94 & $>99$ & $>95: 5$ \\
\hline $\mathbf{n}$ & $4-\mathrm{Cl}$ & $\mathrm{H}$ & $\mathrm{Me}$ & 0 & 89 & 93 & $>95: 5$ \\
\hline 0 & $4-t-\mathrm{Bu}$ & $\mathrm{H}$ & $\mathrm{Me}$ & 0 & 91 & 94 & $>95: 5$ \\
\hline$p$ & $\mathrm{H}$ & $\mathrm{H}$ & Et & 0 & 91 & 96 & $>95: 5$ \\
\hline$q$ & $\mathrm{H}$ & $\mathrm{H}$ & $\mathrm{Bn}$ & 0 & 89 & 93 & $>95: 5$ \\
\hline$r$ & $\mathrm{H}$ & $\mathrm{H}$ & $\mathrm{Me}$ & $S$ & 95 & 91 & $65: 35$ \\
\hline $\mathbf{s}$ & $\mathrm{H}$ & $\mathrm{H}$ & $\mathrm{Me}$ & $S$ & 93 & 94 & $95: 5$ \\
\hline
\end{tabular}

moiety attached to the $\mathrm{N}$ atom. The best result in terms of enantioselectivity $(85 \%$ ee) was obtained with quinine-derived thiourea in dichloroethane as the solvent. The scope of the reaction was also investigated vis-a-vis the effect of the substitution on the isoxazolinone ring and $p$-quinone methide ( $p$-QM) partner. (Table 15) [51].

Takemoto and co-workers investigated three catalytic systems, namely arylboronic acid alone, its dual combination with chiral thiourea and integrated catalyst having boronic acid functionality in the chiral thiourea molecule. The dual combination of arylboronic acid with chiral thiourea was found as effective as arylboronic acid alone for the intermolecular asymmetric Michael addition of alk-2-enoic acids $\mathbf{6 8}$ with $O$-benzyl- hydroxylamine (69) giving racemic mixture of the product in poor yield. However, the integrated catalyst having boronic acid functionality in the chiral thiourea molecule gave the desired $\beta$-benzyloxyamino acid as the single product in a satisfactory yield. Thus, a series of these catalysts was screened. The best results in term of the yield (83\%) and ee (90\%) were obtained while using the catalyst having a $p$-nitrophenyl group on the other side of thiourea moiety in $\mathrm{CCl}_{4}$ in the presence of $4 \AA$ molecular sieves (Table 16). The yields ranged $57-89 \%$ with ee 70-97\% [52].

A similar chiral multifunctional thiourea/boronic acid was used as an organocatalyst by Michigami et al. for the enantioselective synthesis of $N$-hydroxyaspartic acid derivatives 76 with 
Table 15: Enantioselective 1,6-aza-Michael addition of isoxazolin-5-ones to $p$-quinone methides.<smiles>[R]C1=NOC(=O)C1[R]</smiles>

64<smiles>CC(C)(C)C1=CC(=C[123I])C=C(C(C)(C)C)C1=O</smiles>

65<smiles>C[AsH3]</smiles>

DCE, it<smiles>[R]c1c([R])n(C([Al])c2cc(C(C)(C)C)c(O)c(C(C)(C)C)c2)oc1=O</smiles>

67a-w<smiles>C=CC1CN2CC1CC2C(NC(=S)Nc1cc(C(F)(F)F)cc(C(F)(F)F)c1)c1ccnc2ccc(OC)cc12</smiles>

cat. 66

\begin{tabular}{|c|c|c|c|c|c|}
\hline 67 & $\mathrm{R}^{1}$ & $\mathrm{R}^{2}$ & $\mathrm{Ar}$ & Yield [\%] & ee $[\%]^{a}$ \\
\hline a & $\mathrm{Me}$ & $\mathrm{H}$ & $\mathrm{Ph}$ & 65 & 87 \\
\hline b & $\mathrm{Et}$ & $\mathrm{H}$ & $\mathrm{Ph}$ & 51 & 81 \\
\hline c & $\operatorname{Pr}$ & $\mathrm{H}$ & $\mathrm{Ph}$ & 50 & 81 \\
\hline d & $\mathrm{Ph}$ & $\mathrm{H}$ & $\mathrm{Ph}$ & 77 & 54 \\
\hline e & $\operatorname{Pr}$ & $\mathrm{H}$ & $\mathrm{Ph}$ & 78 & 89 \\
\hline f & $\mathrm{Me}$ & $\mathrm{Me}$ & $\mathrm{Ph}$ & 66 & 62 \\
\hline g & $\mathrm{Me}$ & $\mathrm{H}$ & $p-\mathrm{MeC}_{6} \mathrm{H}_{4}$ & 62 & 88 \\
\hline $\mathbf{h}$ & $\mathrm{Me}$ & $\mathrm{H}$ & $p-\mathrm{MeOC}_{6} \mathrm{H}_{4}$ & 74 & 84 \\
\hline $\mathbf{i}$ & $\mathrm{Me}$ & $\mathrm{H}$ & $p-\mathrm{ClC}_{6} \mathrm{H}_{4}$ & 43 & 48 \\
\hline j & $\mathrm{Me}$ & $\mathrm{H}$ & $p-\mathrm{O}_{2} \mathrm{NC}_{6} \mathrm{H}_{4}$ & 47 & 89 \\
\hline $\mathbf{k}$ & $\mathrm{Me}$ & $\mathrm{H}$ & $o-\mathrm{MeOC}_{6} \mathrm{H}_{4}$ & 81 & 94 \\
\hline $\mathbf{I}$ & $\mathrm{Me}$ & $\mathrm{H}$ & $0-\mathrm{ClC}_{6} \mathrm{H}_{4}$ & 94 & 96 \\
\hline m & $\mathrm{Me}$ & $\mathrm{H}$ & $o-\mathrm{BrC}_{6} \mathrm{H}_{4}$ & 43 & 90 \\
\hline $\mathbf{n}$ & $\mathrm{Me}$ & $\mathrm{H}$ & $m-\mathrm{MeOC}_{6} \mathrm{H}_{4}$ & 20 & 25 \\
\hline 0 & $\mathrm{Me}$ & $\mathrm{H}$ & $m-\mathrm{ClC}_{6} \mathrm{H}_{4}$ & 36 & 81 \\
\hline p & $\mathrm{Me}$ & $\mathrm{H}$ & $m-\mathrm{O}_{2} \mathrm{NC}_{6} \mathrm{H}_{4}$ & 56 & 77 \\
\hline q & $\operatorname{Pr}$ & $\mathrm{H}$ & $p-\mathrm{MeOC}_{6} \mathrm{H}_{4}$ & 75 & 79 \\
\hline $\mathbf{r}$ & $\operatorname{Pr}$ & $\mathrm{H}$ & $p-\mathrm{ClC}_{6} \mathrm{H}_{4}$ & 78 & 88 \\
\hline $\mathbf{s}$ & $\operatorname{Pr}$ & $\mathrm{H}$ & $p-\mathrm{O}_{2} \mathrm{NC}_{6} \mathrm{H}_{4}$ & 80 & 86 \\
\hline$t$ & $\operatorname{Pr}$ & $\mathrm{H}$ & $o-\mathrm{ClC}_{6} \mathrm{H}_{4}$ & 76 & 92 \\
\hline $\mathbf{u}$ & $\operatorname{Pr}$ & $\mathrm{H}$ & $m-\mathrm{MeOC}_{6} \mathrm{H}_{4}$ & 82 & 82 \\
\hline $\mathbf{v}$ & $\operatorname{Pr}$ & $\mathrm{H}$ & $m-\mathrm{ClC}_{6} \mathrm{H}_{4}$ & 80 & 88 \\
\hline w & $\operatorname{Pr}$ & $\mathrm{H}$ & $\mathrm{Ph}$ & 71 & 86 \\
\hline
\end{tabular}

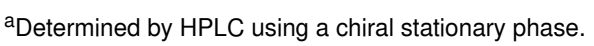

perfect regioselectivity and high enantioselectivity (Table 17) [53].

Likewise, Miyaji et al. reported an efficient method for the synthesis of 2-substituted indolines $\mathbf{7 9}$ via intramolecular azaMichael addition of $\alpha, \beta$-unsaturated carboxylic acid derivatives 77 in the presence of bifunctional thiourea organocatalysts (cat.
78) (Table 18). The product was obtained in moderate to good yield of $53-99 \%$ with an ee of $74-93 \%$ [54].

Liu et al. accomplished a catalytic cascade aza-Michael-Henrydehydration protocol for the preparation of chiral 3-nitro-1,2dihydroquinolines $\mathbf{8 3}$ from the reaction of $\mathrm{N}$-protected aminobenzaldehydes $\mathbf{8 0}$ with substituted nitroolefins $\mathbf{8 1}$ by using 
Table 16: Asymmetric intermolecular aza-Michael addition of $(E)$-3-substituted-2-enoic acid

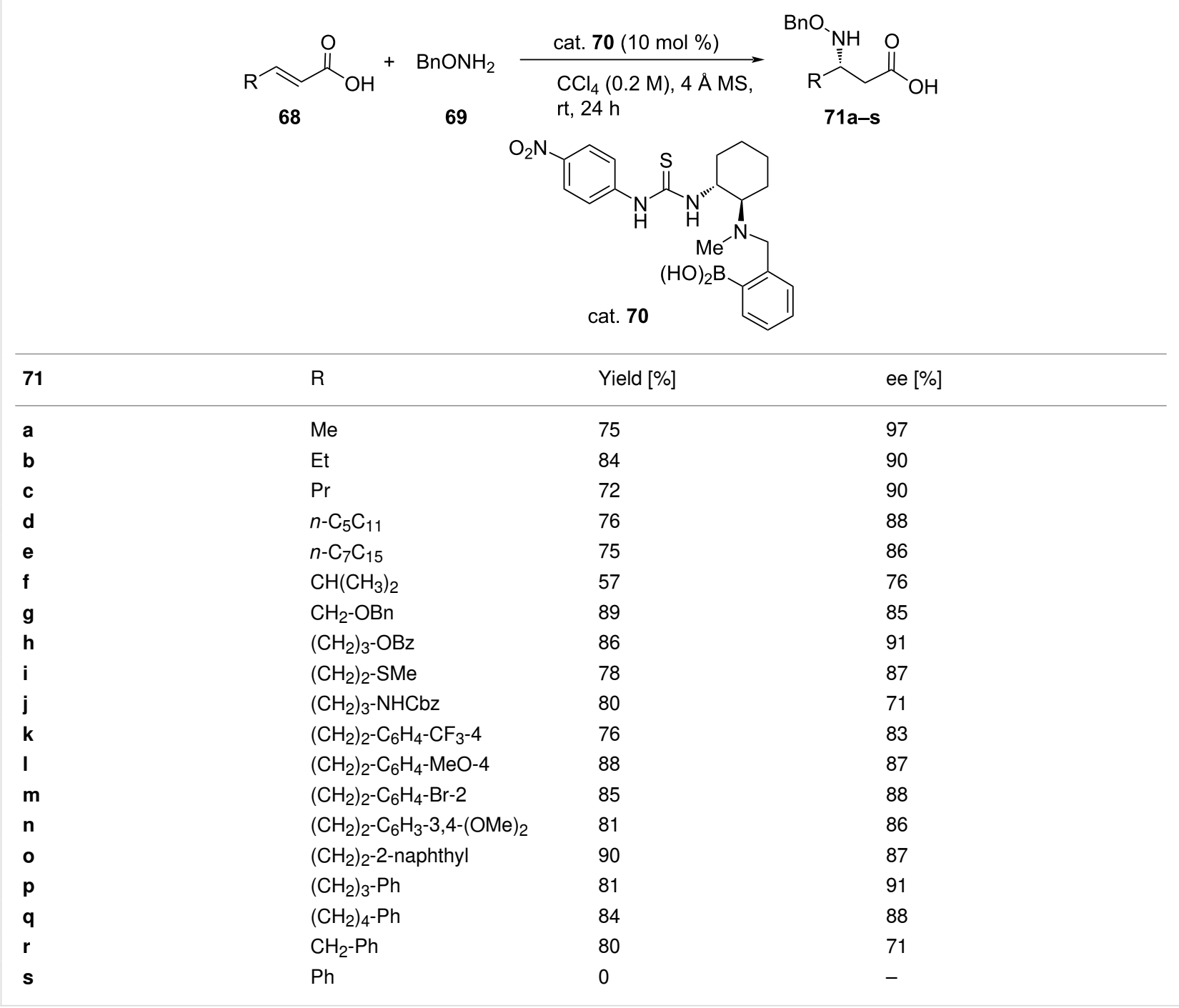

Table 17: Asymmetric aza-Michael addition reaction for the synthesis of $N$-hydroxyaspartic acid derivatives catalyzed by chiral multifunctional thiourea/boronic acid.<smiles>[X]C(=O)C=CC(=O)O</smiles>

$+$

$\mathrm{BnONH}_{2}$

73 cat. $74(10 \mathrm{~mol} \%)$

$\mathrm{PhCO}_{2} \mathrm{H}$ (1 equiv), $4 \AA \mathrm{MS}, \mathrm{CCl}_{4}$, rt then $\mathrm{TMSCHN}_{2}$

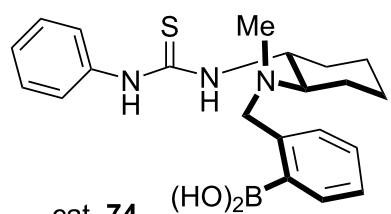<smiles>[R]C(=O)OC(=O)C[C@H](NOCc1ccccc1)C([X])=O</smiles>

75<smiles>[R]C(=O)N[C@@H](CCOC)C([X])=O</smiles>

76a-g

$$
\mathrm{R}^{\prime}=\underset{\mathrm{FmocHNCH}}{\mathrm{PhCH}_{2} \mathrm{CH}_{2},}
$$

\begin{tabular}{lclll}
\hline 76 & $\mathrm{X}$ & Yield [\%] & ee [\%] & $\mathrm{dr}$ \\
\hline a & $t$-BuO & 88 & 93 & -
\end{tabular}


Table 17: Asymmetric aza-Michael addition reaction for the synthesis of $N$-hydroxyaspartic acid derivatives catalyzed by chiral multifunctional thiourea/boronic acid. (continued)

\begin{tabular}{|c|c|c|c|c|}
\hline b & $\mathrm{BnO}$ & 50 & 91 & - \\
\hline C & $\mathrm{EtO}$ & 49 & 94 & - \\
\hline d & & 40 & - & $67: 33$ \\
\hline e & & 60 & - & $75: 25$ \\
\hline $\mathbf{f}$ & & 66 & 63 & - \\
\hline g & & 81 & 85 & $73: 37$ \\
\hline
\end{tabular}

Table 18: Intramolecular aza-Michael addition catalyzed by bifunctional thiourea.<smiles>[R]C(=O)/C=C\Cc1cc([R])ccc1NC(=O)OCc1ccccc1</smiles>

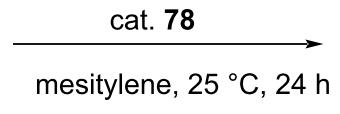

77<smiles>C=CC1CN2CCC1C2[C@H](NC(=O)Nc1cc(C(F)(F)F)cc(C(F)(F)F)c1)c1ccnc2ccc(OC)cc12</smiles>

cat. 78<smiles>[R1]C(=O)C[C@H]1Cc2cc([R])ccc2N1C([R])=O</smiles>

79a-j

\begin{tabular}{lllll}
\hline $\mathbf{7 9}$ & $\mathrm{R}^{1}$ & $\mathrm{R}^{2}$ & Yield [\%] & ee [\%] \\
\hline $\mathbf{a}$ & $\mathrm{Ph}$ & $\mathrm{H}$ & 99 & 87 \\
$\mathbf{b}$ & $4-\mathrm{CH}_{3} \mathrm{OC}_{6} \mathrm{H}_{4}$ & $\mathrm{H}$ & 73 & 84 \\
$\mathbf{c}$ & $4-\mathrm{CF}_{3} \mathrm{C}_{6} \mathrm{H}_{4}$ & $\mathrm{H}$ & 79 & 88 \\
$\mathbf{d}$ & $2-\mathrm{naphthyl}_{\mathbf{m}}$ & $\mathrm{H}$ & 83 & 88 \\
$\mathbf{e}$ & $4-\mathrm{BrC}_{6} \mathrm{H}_{4}$ & $\mathrm{H}$ & 75 & 91 \\
$\mathbf{f}$ & $\mathrm{Ph}$ & $\mathrm{CH}_{3} \mathrm{O}$ & 82 & 83 \\
$\mathbf{g}$ & $\mathrm{Ph}$ & $\mathrm{F}$ & 69 & 82 \\
$\mathbf{h}$ & $\mathrm{Ph}$ & $\mathrm{Cl}$ & 82 & 84 \\
$\mathbf{i}$ & $4-\mathrm{BrC}_{6} \mathrm{H}_{4}$ & $\mathrm{CH}_{3} \mathrm{O}$ & 53 & 93 \\
$\mathbf{j}$ & $\mathrm{CH}_{3}$ & $\mathrm{H}$ & 18 & 74
\end{tabular}

tertiary amine-thiourea catalyst (cat. 82). This cascade reaction afforded aza-Michael adducts in $77-92 \%$ yields with high ee (up to $90 \%$ ) (Table 19) [55].
Du et al. developed an enantioselective catalytic tandem aminolysis/aza-Michael addition for the asymmetric total synthesis of two natural Apocynaceae alkaloids, (+)-deethylibophyllidine 
Table 19: Intramolecular aza-Michael addition reaction catalyzed by tertiary amine-thiourea.

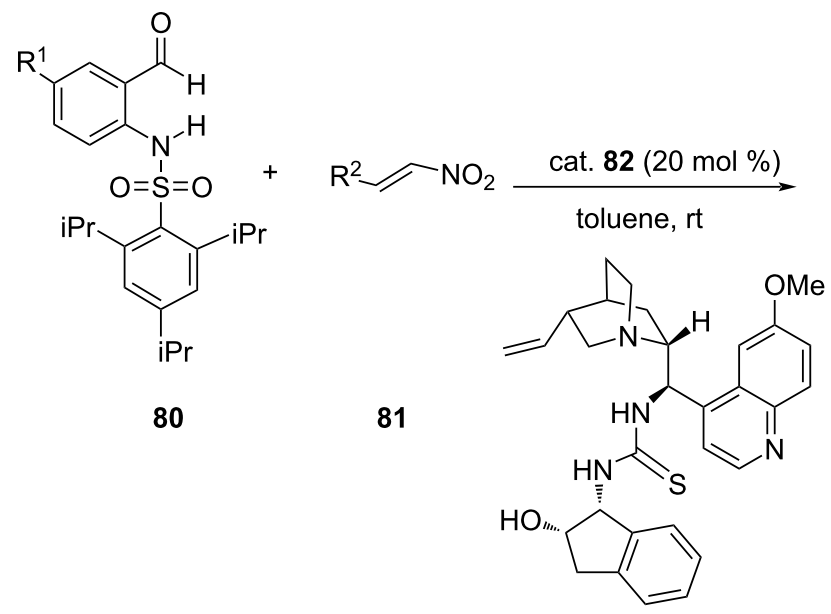<smiles>[R]c1ccc2c(c1)C=C([N+](=O)[O-])C([R])N2</smiles><smiles>CC(C)c1cc(C(C)C)c(S(C)(=O)=O)c(C(C)C)c1</smiles>

$83 a-k$

cat. 82

\begin{tabular}{|c|c|c|c|c|}
\hline 83 & $\mathrm{R}^{1}$ & $\mathrm{R}^{2}$ & Yield [\%] & ee [\%] \\
\hline $\mathbf{a}$ & $\mathrm{H}$ & $\mathrm{Ph}$ & 81 & 90 \\
\hline b & $\mathrm{H}$ & $4-\mathrm{FC}_{6} \mathrm{H}_{4}$ & 77 & 82 \\
\hline c & $\mathrm{H}$ & $4-\mathrm{BrC}_{6} \mathrm{H}_{4}$ & 91 & 85 \\
\hline d & $\mathrm{H}$ & $4-\mathrm{NCC}_{6} \mathrm{H}_{4}$ & 92 & 87 \\
\hline e & $\mathrm{H}$ & 4- $\mathrm{MeC}_{6} \mathrm{H}_{4}$ & 83 & 81 \\
\hline$f$ & $\mathrm{H}$ & 4- $\mathrm{MeOC}_{6} \mathrm{H}_{4}$ & 75 & 84 \\
\hline g & $\mathrm{H}$ & $3-\mathrm{ClC}_{6} \mathrm{H}_{4}$ & 90 & 87 \\
\hline $\mathbf{h}$ & $\mathrm{H}$ & $3-\mathrm{BrC}_{6} \mathrm{H}_{4}$ & 86 & 89 \\
\hline i & $\mathrm{H}$ & $3-\mathrm{MeC}_{6} \mathrm{H}_{4}$ & 83 & 81 \\
\hline j & $\mathrm{H}$ & $\mathrm{CH}(\mathrm{Me})_{2}$ & 86 & 70 \\
\hline $\mathbf{k}$ & $\mathrm{Cl}$ & $\mathrm{Ph}$ & 78 & 88 \\
\hline
\end{tabular}

$\mathbf{( 8 8 )}$ and (+)-limaspermidine $(\mathbf{8 9})$ from the reaction of paradienone imide 84 with benzylamine $(\mathbf{8 5})$ in the presence of bifunctional thiourea organocatalyst (Scheme 4) [56].

\subsection{Reactions catalyzed by chiral binol-derived phosphoric acids}

Binol-derived chiral phosphoric acids have been shown to catalyze the reactions via single or double hydrogen bonding $[57,58]$.

Saito et al. accomplished the chiral phosphoric acid-catalyzed intramolecular aza-Michael addition reaction of $\mathrm{N}$-unprotected 2-aminophenyl vinyl ketones $\mathbf{9 0}$ to obtain chiral 2-substituted 2,3-dihydro-4-quinolones 92 in very good yields (67-95\%) with high ee (82-97\%) (Table 20) [59].

Following a similar approach, Yang et al. reported asymmetric aza-Michael additions of anilines 94 to $\beta$-nitrostyrenes 93 using a chiral binol-derived phosphoric acid diester catalyst (cat. 95).
They succeeded in preparing $\beta$-nitroamines 96 in good yields (65-85\%), but with only a moderate level of ee (19-70\%) (Table 21) [60].

Feng et al. accomplished an asymmetric intramolecular azaMichael addition of activated $\alpha, \beta$-unsaturated ketones 97 by using chiral $N$-triflylphosphoramide as catalyst (cat. 98). The products, namely 2-aryl-2,3-dihydro-4-quinolones 99 were obtained in good yields of up to $95 \%$ and good ee (58-72\%) (Table 22) [61].

\section{Covalent-bonding organocatalysis of aza-Michael reactions}

This category of organocatalysts includes N-heterocyclic carbenes and pyrrolidine derivatives.

\subsection{Catalysis by $\mathrm{N}$-heterocyclic carbenes ( $\mathrm{NHC}$ )}

In recent years, NHCs have been used as organocatalysts for a wide variety of reactions [62]. 
<smiles>O=C1C=CC2(C=C1)CC(=O)N(C(=O)OCc1ccccc1)c1ccccc12</smiles>

84<smiles>NCc1ccccc1</smiles>

85
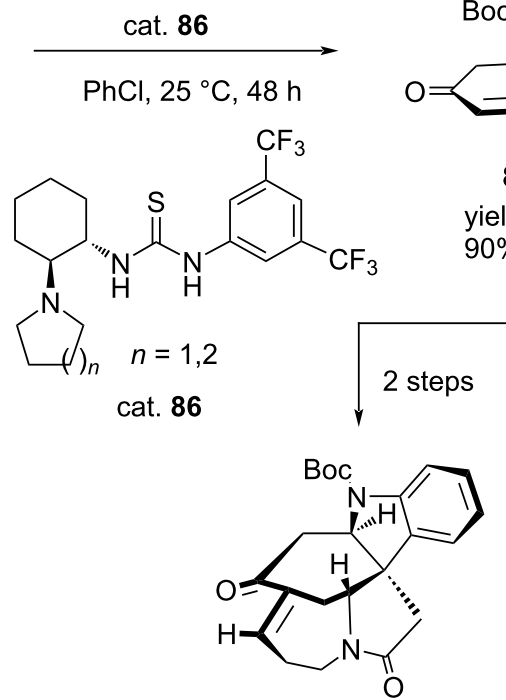

89

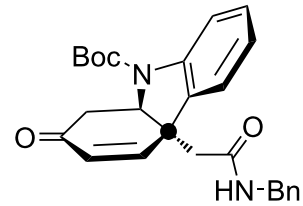

87

yield $45 \%$

$90 \%$ ee

\section{$\mathrm{HN}-\mathrm{Bn}$}
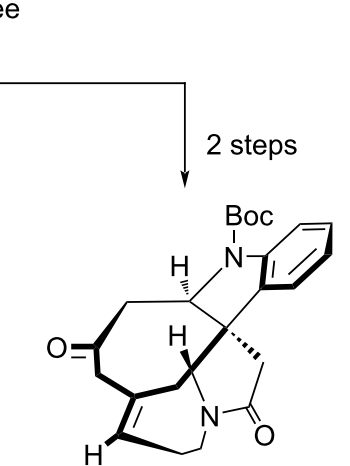

88

$\downarrow$ multiple steps

(+)-limaspermidine yield $93 \%$

$\downarrow$ multiple steps

(+)-deethylibophyllidine yield $34 \%$

Scheme 4: Asymmetric aza-Michael addition of para-dienone imide to benzylamine.

Table 20: Intramolecular aza-Michael addition reaction catalyzed by chiral phosphoric acid.<smiles>[R]C=CC(=O)c1ccccc1N</smiles>

90

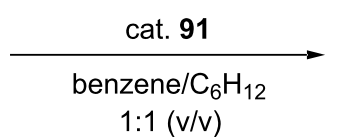

$70{ }^{\circ} \mathrm{C}$<smiles>[X]c1cc2ccccc2c(-c2c(OP(=O)(F)F)c([X])cc3ccccc23)c1OP(=O)(O)O</smiles>

cat. 91<smiles>[R]C1CC(=O)c2ccccc2N1</smiles>

92a-m

\begin{tabular}{llll}
\hline $\mathbf{9 2}$ & $\mathrm{R}$ & Yield [\%] & ee [\%] \\
\hline a & $\mathrm{Ph}$ & 95 & 93 \\
b & $2-\mathrm{FC}_{6} \mathrm{H}_{4}$ & 71 & 90 \\
c & $2-\mathrm{ClC}_{6} \mathrm{H}_{4}$ & 90 & 93 \\
d & $2-\mathrm{BrC}_{6} \mathrm{H}_{4}$ & 90 & 94 \\
e & $2-\mathrm{MeC}_{6} \mathrm{H}_{4}$ & 97 & 88 \\
$\mathbf{f}$ & $3-\mathrm{BrC}_{6} \mathrm{H}_{4}$ & 73 & 84 \\
g & $3-\mathrm{MeC}_{6} \mathrm{H}_{4}$ & 95 & 86
\end{tabular}


Table 20: Intramolecular aza-Michael addition reaction catalyzed by chiral phosphoric acid. (continued)

$\begin{array}{llll}\mathbf{h} & 3-\mathrm{MeOC}_{6} \mathrm{H}_{4} & 95 & 92 \\ \mathbf{i} & 4-\mathrm{FC}_{6} \mathrm{H}_{4} & \text { quant } & 87 \\ \mathbf{j} & 4-\mathrm{ClC}_{6} \mathrm{H}_{4} & 67 & 82 \\ \mathbf{k} & 4-\mathrm{MeC}_{6} \mathrm{H}_{4} & \text { quant } & 97 \\ \mathbf{I} & 2-\text { naphthyl } & 82 & 81 \\ \mathbf{m} & t-\mathrm{Bu} & 64 & 88\end{array}$

Table 21: Asymmetric aza-Michael addition of aniline to $\beta$-nitrostyrenes.

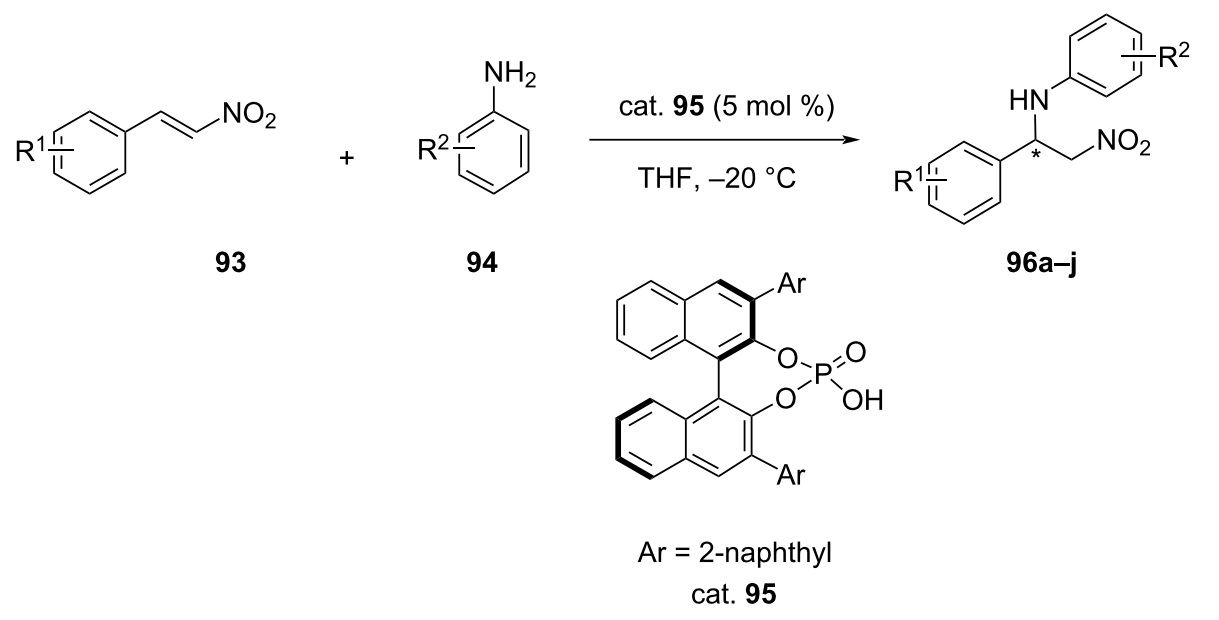

\begin{tabular}{|c|c|c|c|c|}
\hline 96 & $\mathrm{R}^{1}$ & $\mathrm{R}^{2} \frac{r}{11}$ & Yield [\%] & ee [\%] \\
\hline $\mathbf{a}$ & $4-\mathrm{Br}$ & $4-\mathrm{BrC}_{6} \mathrm{H}_{4}$ & 82 & 19 \\
\hline b & $4-\mathrm{Cl}$ & $4-\mathrm{BrC}_{6} \mathrm{H}_{4}$ & 81 & 30 \\
\hline c & 2-OMe & $4-\mathrm{BrC}_{6} \mathrm{H}_{4}$ & 65 & 44 \\
\hline d & 4-OMe & $4-\mathrm{BrC}_{6} \mathrm{H}_{4}$ & - & - \\
\hline e & $2-\mathrm{Br}$ & $4-\mathrm{BrC}_{6} \mathrm{H}_{4}$ & 85 & 45 \\
\hline f & $2,3-(\mathrm{OMe})_{2}$ & $4-\mathrm{BrC}_{6} \mathrm{H}_{4}$ & 85 & 70 \\
\hline g & 4-Me & $\mathrm{Ph}$ & 70 & 30 \\
\hline h & 4-Me & 4- $\mathrm{MeC}_{6} \mathrm{H}_{4}$ & 75 & 30 \\
\hline i & 4-Me & 4- $\mathrm{MeC}_{6} \mathrm{H}_{4}$ & 70 & 42 \\
\hline j & 4-Me & 2-naphthyl & 64 & 48 \\
\hline
\end{tabular}

Wang et al. investigated the use of several 1,2,4-triazoloannelated chiral NHCs as organocatalysts to catalyze enantioselective aza-MR between primary amines (100) and $\beta$-trifluoromethyl- $\beta$-arylnitroolefins $\mathbf{1 0 1}$ and the best results (yield 99\%, ee $91 \%$ ) were obtained in the reaction of benzylamine $\left(\mathrm{R}^{1}=\mathrm{Ph}\right)$ on using the NHC precursor as shown below in the presence of hexafluoroisopropanol (HFIP) as additive along with molecular sieves (4 $\AA$ ) (Table 23) [63]. The role of HFIP is to act as proton shuttle, i.e., to assist in 1,3-prototropic shift.

\subsection{Catalysis by chiral pyrrolidine derivatives}

Chiral pyrrolidine derivatives, such as $(S)$-proline are widely used as organocatalysts $[54,64]$.

Lee et al. synthesized bromopyrrole alkaloids 107 via azaMichael addition of 4,5-dibromo- $1 H$-pyrrole-2-carbonitrile 104 to Bz-protected $(E)$-4-hydroxybut-2-enal $\mathbf{1 0 5}$ in the presence of (S)- $\alpha, \alpha$-bis [3,5-bis(trifluoromethyl)phenyl]-2-pyrrolidinemethanol trimethylsilyl ether as the organocatalyst (cat. 
Table 22: Intramolecular aza-Michael addition reaction catalyzed by chiral $N$-triflylphosphoramide.<smiles>[R]C=C(C(=O)Nc1ccccc1)C(=O)OCc1ccccc1</smiles>

97

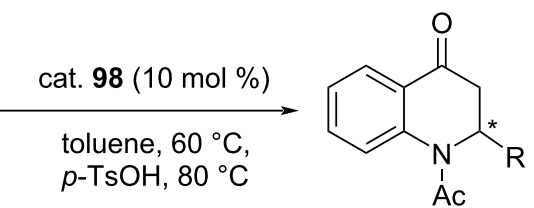

99a-i<smiles>[R]c1cc2ccccc2c(-c2c([R])cc3ccccc3c2OP(N)(=O)[IH2])c1[R]</smiles>

(S)

$\mathrm{R}^{\prime}$ = 1-naphthyl cat. 98

\begin{tabular}{llll}
\hline $\mathbf{9 9}$ & $\mathrm{R}$ & Yield [\%] & ee [\%] \\
\hline $\mathbf{a}$ & $\mathrm{Ph}$ & 95 & $97(-)$ \\
$\mathbf{b}$ & $4-\mathrm{BrC}_{6} \mathrm{H}_{4}$ & 90 & $58(-)$ \\
$\mathbf{c}$ & $4-\mathrm{ClC}_{6} \mathrm{H}_{4}$ & 90 & $67(-)$ \\
$\mathbf{d}$ & $4-\mathrm{NO}_{2} \mathrm{C}_{6} \mathrm{H}_{4}$ & 77 & $20(-)$ \\
$\mathbf{e}$ & $4-\mathrm{MeC}_{6} \mathrm{H}_{4}$ & 98 & $82(-)$ \\
$\mathbf{f}$ & $4-\mathrm{MeOC}_{6} \mathrm{H}_{4}$ & 94 & $60(-)$ \\
$\mathbf{g}$ & $2-\mathrm{MeOC}_{6} \mathrm{H}_{4}$ & 95 & $4(+)$ \\
$\mathbf{h}$ & $1-$ naphthyl & 81 & $76(+)$ \\
$\mathbf{i}$ & $2-$ naphthyl & 98 & $76(-)$
\end{tabular}

Table 23: Aza-Michael addition of primary amines to $\beta$-trifluromethyl- $\beta$-phenylnitroolefin catalyzed nitrogen heterocyclic carbene.

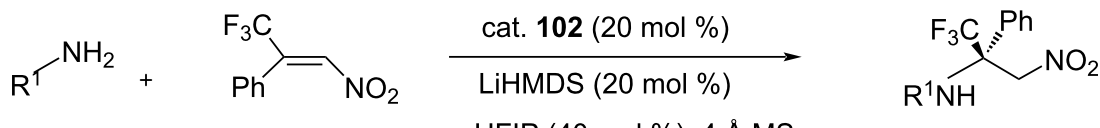

$$
\begin{aligned}
& \text { 100a-q } 101 \text { toluene, }-78^{\circ} \mathrm{C} \quad 103 a-q \\
& \underbrace{O N}_{A r=M e s}
\end{aligned}
$$

\begin{tabular}{|c|c|c|c|}
\hline 103 & $\mathrm{R}^{1}$ & Yield [\%] & ee $[\%]$ \\
\hline a & $\mathrm{C}_{6} \mathrm{H}_{5} \mathrm{CH}_{2-}^{-}$ & 90 & 91 \\
\hline b & $2-\mathrm{MeC}_{6} \mathrm{H}_{4} \mathrm{CH}_{2}^{-}$ & 67 & 91 \\
\hline c & $4-\mathrm{MeOC}_{6} \mathrm{H}_{4} \mathrm{CH}_{2-}^{-}$ & 89 & 92 \\
\hline d & $3,5-(\mathrm{MeO})_{2} \mathrm{C}_{6} \mathrm{H}_{3} \mathrm{CH}_{2^{-}}$ & 56 & 86 \\
\hline e & $\mathrm{C}_{6} \mathrm{H}_{5}\left(\mathrm{CH}_{2}\right)_{2^{-}}^{-}$ & 85 & 86 \\
\hline f & $4-\mathrm{BrC}_{6} \mathrm{H}_{4}\left(\mathrm{CH}_{2}\right)_{2}^{-}$ & 68 & 87 \\
\hline g & $\mathrm{CH}_{3}\left(\mathrm{CH}_{2}\right)_{2-}^{-}$ & 80 & 92 \\
\hline
\end{tabular}

cat. 102 
Table 23: Aza-Michael addition of primary amines to $\beta$-trifluromethyl- $\beta$-phenylnitroolefin catalyzed nitrogen heterocyclic carbene. (continued)

$\begin{array}{llll}\mathbf{h} & \mathrm{C}_{7} \mathrm{H}_{15} \mathrm{CH}_{2-} & 87 & 95 \\ \mathbf{i} & \mathrm{C}_{6} \mathrm{H}_{5}\left(\mathrm{CH}_{2}\right)_{3}^{-} & 82 & 93 \\ \mathbf{j} & \left(\mathrm{CH}_{3}\right)_{2} \mathrm{CH}\left(\mathrm{CH}_{2}\right)_{2}- & 79 & 89 \\ \mathbf{k} & \text { cyclopropyl- } & 79 & 97 \\ \mathbf{~ I} & \text { cyclobutyl- } & 78 & 94 \\ \mathbf{m} & 2-\text { pyridylethyl- } & 87 & 93 \\ \mathbf{n} & \mathrm{BocHN}\left(\mathrm{CH}_{2}\right)_{2} & 79 & 91 \\ \mathbf{0} & \mathrm{MeO}\left(\mathrm{CH}_{2}\right)_{3}^{-} & 87 & 87 \\ \mathbf{p} & (\mathrm{Me})_{2} \mathrm{~N}\left(\mathrm{CH}_{2}\right)_{2}^{-} & 99 & 91 \\ \mathbf{q} & 2 \text { 2-thienyl }\left(\mathrm{CH}_{2}\right)_{2}^{-} & 98 & 93\end{array}$

106) and using $\mathrm{PhCO}_{2} \mathrm{H}$ as the acid additive. Desired products were obtained in good yields $\approx 78 \%$ with excellent enantioselectivities of up to $93 \%$ (Table 24) [65]. The role of the additive is to assist in the formation of the iminium intermediate from the reaction of pyrrolidine with the aldehyde group.

Following a similar approach, Guo et al. accomplished the first organocatalytic asymmetric aza-Michael addition of purine bases 108 to aliphatic $\alpha, \beta$-unsaturated aldehydes 109 and synthesized biologically active acyclonucleoside $\mathbf{1 1 0}$ via an iminium-ion activation mechanism. The initially formed prod- uct was reduced in situ to afford the final product in $82-89 \%$ yield and $89-96 \%$ ee (Table 25) [66].

In a similar method, Joie et al. accomplished an asymmetric organocatalytic quadruple cascade reaction of various $\alpha$-ketoamides 111 with aromatic $\alpha, \beta$-unsaturated aldehydes 112 to obtain tetraaryl-substituted 2-azabicyclo[3.3.0]octadienones 114 in good yields (34-71\%) with excellent diastereo- and enantioselectivities (84-97\%). The reaction occurred via an azaMichael/aldol condensation/vinylogous Michael addition/aldol condensation sequence (Table 26) [67].

Table 24: Asymmetric aza-Michael additions of pyrroles to protected $(E)$-4-hydroxybut-2-enals.<smiles>[R]c1cc(Br)c(Br)[nH]1</smiles>

104<smiles>[R]OC/C=C/C=O</smiles>

105 i) cat. $106(20 \mathrm{~mol} \%)$

$\mathrm{PhCO}_{2} \mathrm{H}(40 \mathrm{~mol} \%)$ toluene, $-20^{\circ} \mathrm{C}, 18 \mathrm{~h}$ ii) $\mathrm{NaBH}_{4}(110 \mathrm{~mol} \%)$ EtOH $(0.1 \mathrm{~mol} \%),-20{ }^{\circ} \mathrm{C}$ $5 \mathrm{~h}$<smiles>[Mg]OC(Br)(Br)C1CCCN1</smiles>
$\mathrm{Ar}=3,5-\left(\mathrm{CF}_{3}\right)_{2} \mathrm{C}_{6} \mathrm{H}_{3}$ cat. 106

\begin{tabular}{lllll}
\hline $\mathbf{1 0 7}$ & $\mathrm{R}^{1}$ & $\mathrm{R}^{2}$ & Yield [\%] & ee [\%] \\
\hline $\mathbf{a}$ & $\mathrm{CN}$ & $\mathrm{Bz}$ & 70 & 76 \\
$\mathbf{b}$ & $\mathrm{CO}_{2} \mathrm{CH}_{3}$ & $\mathrm{Bz}$ & n.d. ${ }^{\mathrm{a}}$ & - \\
$\mathbf{c}$ & $\mathrm{CN}$ & TBS & 78 & 80 \\
$\mathbf{d}$ & $\mathrm{CN}$ & TBDPS & 77 & 87 \\
$\mathbf{e}$ & $\mathrm{CN}$ & TBDPS & 76 & 91 \\
$\mathbf{f}$ & $\mathrm{CN}$ & TBDPS & 76 & 93 \\
\hline
\end{tabular}

aNot determined. 
Table 25: Asymmetric aza-Michael addition of purine bases to aliphatic $\alpha, \beta$-unsaturated aldehydes.<smiles>Clc1nc(Cl)c2nc[nH]c2n1</smiles>

108<smiles>[R]C=CC=O</smiles>

109 cat. $106(10 \mathrm{~mol} \%)$

$\mathrm{PhCO}_{2} \mathrm{H}(10 \mathrm{~mol} \%)$ toluene, $-30^{\circ} \mathrm{C}$ $\mathrm{NaBH}_{4}, \mathrm{MeOH}, 0^{\circ} \mathrm{C}$<smiles>[R]C(CCO)n1cnc2c(Cl)nc(Cl)nc21</smiles>

$110 \mathrm{a}-\mathrm{h}$

\begin{tabular}{llll}
\hline $\mathbf{1 1 0}$ & $\mathrm{R}$ & Yield [\%] & ee [\%] \\
\hline $\mathbf{a}$ & $\mathrm{Me}$ & 87 & 89 \\
$\mathbf{b}$ & $\mathrm{Et}$ & 89 & 91 \\
$\mathbf{c}$ & $n-\mathrm{Pr}$ & 87 & 96 \\
$\mathbf{d}$ & $n$-Bu & 86 & 94 \\
$\mathbf{e}$ & $n$-pentyl & 85 & 96 \\
$\mathbf{f}$ & $n$-hexyl & 87 & 96 \\
$\mathbf{g}$ & $\mathrm{CH}$ OTBS & 82 & 96 \\
$\mathbf{h}$ & $\mathrm{Ph}$ & trace & -
\end{tabular}

Table 26: Asymmetric aza-Michael organocatalytic quadruple cascade reaction.<smiles>[R][CH-]C(C=O)=CC(=O)OCC</smiles>

111

112<smiles>COC(c1ccccc1)(c1ccccc1)C1CCCN1</smiles><smiles>[R]C1=C2C=C(C)[C@@H]([R])[C@]2([R1])NC(=O)C1=O</smiles>

$114 a-i$

cat. 113

\begin{tabular}{llllll}
\hline $\mathbf{1 1 4}$ & $\mathrm{R}^{1}$ & $\mathrm{R}^{2}$ & $\mathrm{R}^{3}$ & Yield [\%] $^{\text {ee [\%] }}$ \\
\hline $\mathbf{a}$ & $\mathrm{Ph}$ & $\mathrm{Ph}$ & $\mathrm{Ph}$ & 63 & 97 \\
$\mathbf{b}$ & $\mathrm{Ph}$ & $\mathrm{Ph}$ & $4-\mathrm{MeOC}_{6} \mathrm{H}_{4}$ & 51 & $89(91)$ \\
$\mathbf{c}$ & $\mathrm{Ph}$ & $\mathrm{Ph}$ & $4-\mathrm{ClC}_{6} \mathrm{H}_{4}$ & 34 & $85(95)$ \\
$\mathbf{d}$ & $\mathrm{Ph}$ & $\mathrm{Ph}$ & $2,3-\left(\mathrm{OCH}_{2} \mathrm{O}\right) \mathrm{C}_{6} \mathrm{H}_{3}$ & 56 & $84(87)$ \\
$\mathbf{e}$ & $4-\mathrm{MeOC}_{6} \mathrm{H}_{4}$ & $\mathrm{Ph}$ & $\mathrm{Ph}$ & 66 & $92(91)$ \\
$\mathbf{f}$ & $3-\mathrm{ClC}_{6} \mathrm{H}_{4}$ & $\mathrm{Ph}$ & $\mathrm{Ph}$ & 69 & $91(95)$ \\
$\mathbf{g}$ & $4-\mathrm{O}_{2} \mathrm{NC}_{6} \mathrm{H}_{4}$ & $\mathrm{Ph}$ & $\mathrm{Ph}$ & 58 & 95 \\
$\mathbf{h}$ & $\mathrm{Ph}$ & $4-\mathrm{MeC}_{6} \mathrm{H}_{4}$ & $\mathrm{Ph}$ & 70 & 88 \\
$\mathbf{i}$ & $\mathrm{Ph}$ & $4-\mathrm{ClC}_{6} \mathrm{H}_{4}$ & $\mathrm{Ph}$ & 71 & 95 \\
\hline
\end{tabular}

aValues in brackets correspond to the results obtained with the catalyst $(R)-\mathbf{1 1 3}$.

Recently, the synthesis of axially chiral 4-naphthylquinoline-3carbaldehydes 117 has been reported via Michael/Aldol cascade reaction of alkynals 116 with $N$-(2-(1-naphthoyl)phenyl)benzenesulfonamides $\mathbf{1 1 5}$ using the same pyrrolidine catalyst 113. The products were obtained in excellent yields and enantioselectivities (Table 27) [68]. In this context, the presence of a strong electron-withdrawing sulfonyl group was found to be essential. On comparing efficacies of different sulfonyl 
Table 27: Asymmetric synthesis of chiral 4-naphthylquinoline-3-carbaldehydes.

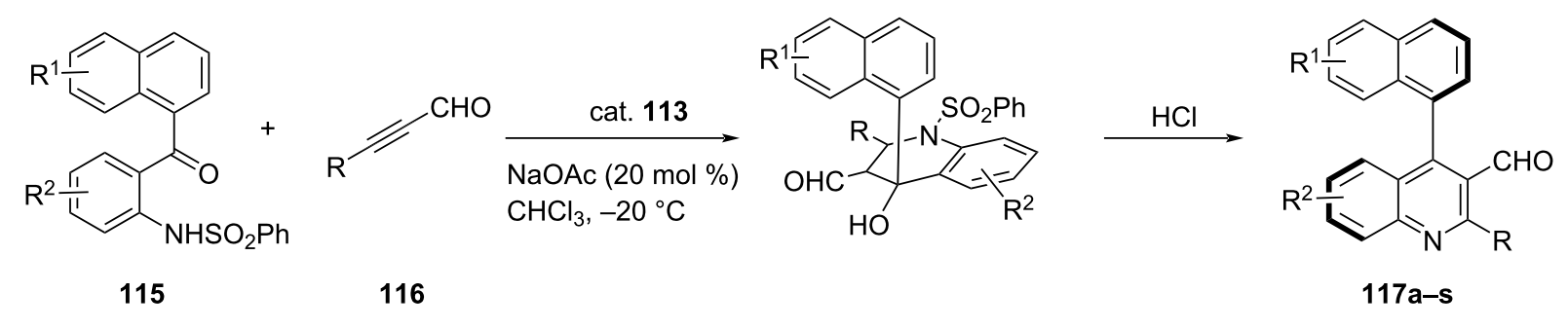

\begin{tabular}{|c|c|c|c|c|c|}
\hline 117 & $\mathrm{R}^{1}$ & $\mathrm{R}^{2}$ & $\mathrm{R}$ & Yield [\%] & ee [\%] \\
\hline $\mathbf{a}$ & $\mathrm{H}$ & $\mathrm{H}$ & $4-\mathrm{ClC}_{6} \mathrm{H}_{4}$ & 97 & 93 \\
\hline b & $\mathrm{H}$ & $\mathrm{H}$ & $\mathrm{Ph}$ & 94 & 95 \\
\hline C & $\mathrm{H}$ & $\mathrm{H}$ & $4-\mathrm{FC}_{6} \mathrm{H}_{4}$ & 86 & 94 \\
\hline d & $\mathrm{H}$ & $\mathrm{H}$ & 4- $\mathrm{BrC}_{6} \mathrm{H}_{4}$ & 95 & 94 \\
\hline e & $\mathrm{H}$ & $\mathrm{H}$ & 4- $\mathrm{MeC}_{6} \mathrm{H}_{4}$ & 81 & 94 \\
\hline f & $\mathrm{H}$ & $\mathrm{H}$ & 4- $\mathrm{MeOC}_{6} \mathrm{H}_{4}$ & 86 & 95 \\
\hline $\mathbf{g}$ & $\mathrm{H}$ & $\mathrm{H}$ & $3-\mathrm{ClC}_{6} \mathrm{H}_{4}$ & 92 & 91 \\
\hline $\mathbf{h}$ & $\mathrm{H}$ & $\mathrm{H}$ & $3-\mathrm{MeC}_{6} \mathrm{H}_{4}$ & 88 & 93 \\
\hline i & $\mathrm{H}$ & $\mathrm{H}$ & 3- $\mathrm{MeOC}_{6} \mathrm{H}_{4}$ & 83 & 93 \\
\hline j & $\mathrm{H}$ & $\mathrm{H}$ & $n-\mathrm{C}_{5} \mathrm{H}_{11}$ & 85 & 87 \\
\hline k & 4-Me & $\mathrm{H}$ & $4-\mathrm{ClC}_{6} \mathrm{H}_{4}$ & 82 & 92 \\
\hline I & 4-MeO & $\mathrm{H}$ & $4-\mathrm{ClC}_{6} \mathrm{H}_{4}$ & 91 & 90 \\
\hline m & $4-\mathrm{F}$ & $\mathrm{H}$ & $4-\mathrm{ClC}_{6} \mathrm{H}_{4}$ & 83 & 94 \\
\hline $\mathbf{n}$ & $\mathrm{H}$ & $6-\mathrm{Cl}$ & $4-\mathrm{ClC}_{6} \mathrm{H}_{4}$ & 90 & 94 \\
\hline 0 & $\mathrm{H}$ & $7-\mathrm{Cl}$ & $4-\mathrm{ClC}_{6} \mathrm{H}_{4}$ & 91 & 91 \\
\hline $\mathbf{p}$ & $\mathrm{H}$ & $7-\mathrm{Cl}$ & $\mathrm{Ph}$ & 95 & 96 \\
\hline $\mathbf{q}$ & $\mathrm{H}$ & $7-\mathrm{Cl}$ & $4-\mathrm{BrC}_{6} \mathrm{H}_{4}$ & 95 & 94 \\
\hline $\mathbf{r}$ & $\mathrm{H}$ & $7-\mathrm{Cl}$ & $3-\mathrm{MeC}_{6} \mathrm{H}_{4}$ & 90 & 95 \\
\hline s & $\mathrm{H}$ & $7-\mathrm{Cl}$ & $n-\mathrm{C}_{5} \mathrm{H}_{11}$ & 88 & 90 \\
\hline
\end{tabular}

groups, benzenesulfonyl moieties with electron-donating groups were found to be most effective. Furthermore, the utility of the newly developed method was demonstrated by preparing useful chiral 4-naphthylquinolines from the resulting products [68].
Chang-Jiang et al. developed a catalytic strategy by using a combination of prolinol silyl ether (cat. 120) and benzoic acid (A1) catalysts to bring about reaction between 3-formyl-substituted indoles or pyrroles 118 and diverse electrophiles, including carbonyls, imines and other Michael acceptors (Scheme 5)<smiles>O=Cc1c[nH]c2ccccc12</smiles><smiles>O=C(/C=C/C(F)(F)F)c1ccccc1</smiles>
cat. $120(20 \mathrm{~mol} \%)$

A1 $(20 \mathrm{~mol} \%)$ toluene, $40{ }^{\circ} \mathrm{C}, 24 \mathrm{~h}$

118
119<smiles>O=Cc1cn(C(CC(=O)c2ccccc2)C(F)(F)F)c2ccccc12</smiles>

121

yield up to $98 \%$ $13 \%$ ee

cat. 120 
[69]. The reaction with secondary amines occurred via the formation of HOMO raised dearomative aza-dienamine-type intermediates, which undergo direct aza-Michael addition to $\beta$-trifluoromethyl enones to afford $N$-functionalized heteroarenes 121 efficiently in moderate to excellent yields, albeit with low to fair enantioselectivity. However, asymmetric aza-Michael additions of these heteroarenes with crotonaldehyde yielded the adducts in moderate to good enantioselectivity under dual catalysis of chiral amines (Scheme 5) [69].

\section{Conclusion}

The asymmetric aza-Michael reaction being a useful synthetic strategy for constructing $\mathrm{C}-\mathrm{N}$ bonds to make a variety of nitrogen-containing chiral scaffolds of wide applications in the fields of pharmaceuticals, organic synthesis building blocks and accessible catalysis continues to attract attention of the chemists. During the last two decades, many new chiral organocatalysts have been developed for accomplishing these reactions with the nitrogen nucleophiles, such as aromatic amines and amides which are otherwise averse to reacting. The organocatalysts have emerged as catalysts of choice due to various reasons, such as their compatibility with the 'Green Chemistry' and possibility of tailoring them according to the requirements. Efforts are directed towards enhancing not only the yields of the products but also enantio- and diastereoselectivities of the azaMichael reactions. New strategies have been adopted while making optimum utilization of the efficacies of the catalysts. Of these strategies, cascade reactions of the Michael addition in conjunction with one or more reactions leading to overall very high yields and ee are noteworthy. Another strategy of interest appears to be the generation of organocatalysts of enhanced efficacy in situ by mixing squaramides with amino acids again giving $>99 \%$ ee. It may be perceived that in the coming years, more sophisticated methodologies will be developed with the advent of new organocatalysts to accomplish asymmetric azaMichael reactions of even the so far unexplored and obstinate amines and amides substrates.

\section{Funding}

The authors express gratitude to the authorities of the IIS (deemed to be University), Jaipur, India for providing the necessary research facilities.

\section{ORCID ${ }^{\circledR}$ iDs}

Raakhi Gupta - https://orcid.org/0000-0003-0732-5134 Raj K. Bansal - https://orcid.org/0000-0002-8154-9817

\section{References}

1. Michael, A. J. Prakt. Chem. 1887, 35, 349-356. doi:10.1002/prac. 18870350136
2. Michael, A. J. Prakt. Chem. 1894, 49, 20-25. doi:10.1002/prac.18940490103

3. Krause, N.; Hoffmann-Röder, A. Synthesis 2001, 171-196. doi:10.1055/s-2001-10803

4. Sibi, M. P.; Manyem, S. Tetrahedron 2000, 56, 8033-8061. doi:10.1016/s0040-4020(00)00618-9

5. Leonard, J.; DíezBarra, E.; Merino, S. Eur. J. Org. Chem. 1998, 2051-2061.

doi:10.1002/(sici)1099-0690(199810)1998:10<2051::aid-ejoc2051>3.0. $\mathrm{co} ; 2-\mathrm{t}$

6. Rossiter, B. E.; Swingle, N. M. Chem. Rev. 1992, 92, 771-806. doi:10.1021/cr00013a002

7. d'Angelo, J.; Desmaële, D.; Dumas, F.; Guingant, A. Tetrahedron: Asymmetry 1992, 3, 459-505. doi:10.1016/s0957-4166(00)80251-7

8. Bartoli, G.; Cimarelli, C.; Marcantoni, E.; Palmieri, G.; Petrini, M. J. Org. Chem. 1994, 59, 5328-5335. doi:10.1021/jo00097a039

9. Hecht, S. M. Acc. Chem. Res. 1986, 19, 383-391. doi:10.1021/ar00132a002

10. Wang, Y.-F.; Izawa, T.; Kobayashi, S.; Ohno, M. J. Am. Chem. Soc. 1982, 104, 6465-6466. doi:10.1021/ja00387a060

11. Hashiguchi, S.; Kawada, A.; Natsugari, $H$. J. Chem. Soc., Perkin Trans. 1 1991, 2435-2444. doi:10.1039/p19910002435

12. Cardillo, G.; Tomasini, C. Chem. Soc. Rev. 1996, 25, 117-128. doi:10.1039/cs9962500117

13. Eliel, E. L.; He, X.-C. J. Org. Chem. 1990, 55, 2114-2119. doi:10.1021/jo00294a026

14. Hayashi, Y.; Rohde, J. J.; Corey, E. J. J. Am. Chem. Soc. 1996, 118, 5502-5503. doi:10.1021/ja960766s

15. Genov, M.; Dimitrov, V.; Ivanova, V. Tetrahedron: Asymmetry 1997, 8, 3703-3706. doi:10.1016/s0957-4166(97)00534-x

16. Thematic issue on organocatalysis. Chem. Rev. 2007, 107, 5413-5883. doi:10.1021/cr078412e

17. Ahrendt, K. A.; Borths, C. J.; MacMillan, D. W. C. J. Am. Chem. Soc. 2000, 122, 4243-4244. doi:10.1021/ja000092s

18. Sánchez-Roselló, M.; Aceña, J. L.; Simón-Fuentes, A.; del Pozo, C. Chem. Soc. Rev. 2014, 43, 7430-7453. doi:10.1039/c4cs00156g

19. Nayak, S.; Panda, P.; Bhakta, S.; Mishra, S. K.; Mohapatra, S. RSC Adv. 2016, 6, 96154-96175. doi:10.1039/c6ra21191g

20. Bhanja, C.; Jena, S.; Nayak, S.; Mohapatra, S. Beilstein J. Org. Chem. 2012, 8, 1668-1694. doi:10.3762/bjoc.8.191

21. Vinogradov, M. G.; Turova, O. V.; Zlotin, S. G. Org. Biomol. Chem. 2019, 17, 3670-3708. doi:10.1039/c8ob03034k

22. Enders, D.; Wang, C.; Liebich, J. X. Chem. - Eur. J. 2009, 15, 11058-11076. doi:10.1002/chem.200902236

23. Wang, J.; Li, P.; Choy, P. Y.; Chan, A. S. C.; Kwong, F. Y. ChemCatChem 2012, 4, 917-925. doi:10.1002/cctc.201200135

24. Krishna, P. R.; Sreeshailam, A.; Srinivas, R. Tetrahedron 2009, 65, 9657-9672. doi:10.1016/j.tet.2009.08.021

25. Cai, Y.-F.; Li, L.; Luo, M.-X.; Yang, K.-F.; Lai, G.-Q.; Jiang, J.-X.; Xu, L.-W. Chirality 2011, 23, 397-403. doi:10.1002/chir.20940

26. Lee, H.-J.; Cho, C.-W. J. Org. Chem. 2013, 78, 3306-3312. doi:10.1021/j04001614

27. Bae, J.-Y.; Lee, H.-J.; Youn, S.-H.; Kwon, S.-H.; Cho, C.-W. Org. Lett. 2010, 12, 4352-4355. doi:10.1021/ol101811c

28. Erkkilä, A.; Majander, I.; Pihko, P. M. Chem. Rev. 2007, 107, 5416-5470. doi:10.1021/cr068388p 
29. Liu, J.-D.; Chen, Y.-C.; Zhang, G.-B.; Li, Z.-Q.; Chen, P.; Du, J.-Y.; Tu, Y.-Q.; Fan, C.-A. Adv. Synth. Catal. 2011, 353, 2721-2730. doi:10.1002/adsc.201100282

30. Zhai, X.-D.; Yang, Z.-D.; Luo, Z.; Xu, H.-T. Chin. Chem. Lett. 2017, 28 , 1793-1797. doi:10.1016/j.cclet.2017.04.017

31. Cheng, S.; Yu, S. Org. Biomol. Chem. 2014, 12, 8607-8610. doi:10.1039/c4ob01646g

32. Ma, S.; Wu, L.; Liu, M.; Xu, X.; Huang, Y.; Wang, Y. RSC Adv. 2013, 3, 11498-11501. doi:10.1039/c3ra41179f

33. Jakkampudi, S.; Konda, S.; Arman, H.; Zhao, J. C.-G. Adv. Synth. Catal. 2020, 362, 2419-2426. doi:10.1002/adsc.202000227

34. Malerich, J. P.; Hagihara, K.; Rawal, V. H. J. Am. Chem. Soc. 2008, 130, 14416-14417. doi:10.1021/ja805693p

35. Ullah, M. S.; Itsuno, S. ACS Omega 2018, 3, 4573-4582. doi:10.1021/acsomega.8b00398

36. Zhao, B.-L.; Du, D.-M. Asian J. Org. Chem. 2015, 4, 1120-1126. doi:10.1002/ajoc.201500306

37. Yang, W.; He, H.-X.; Gao, Y.; Du, D.-M. Adv. Synth. Catal. 2013, 355 , 3670-3678. doi:10.1002/adsc.201300670

38. Zhou, X.-J.; Zhao, J.-Q.; Chen, X.-M.; Zhuo, J.-R.; Zhang, Y.-P.; Chen, Y.-Z.; Zhang, X.-M.; Xu, X.-Y.; Yuan, W.-C. J. Org. Chem. 2019, 84, 4381-4391. doi:10.1021/acs.joc.9b00401

39. Roy, T. K.; Parhi, B.; Ghorai, P. Angew. Chem., Int. Ed. 2018, 57 , 9397-9401. doi:10.1002/anie.201805020

40. Li, J.-H.; Du, D.-M. Chem. - Asian J. 2014, 9, 3278-3286. doi:10.1002/asia.201402706

41. Rajasekar, S.; Anbarasan, P. Org. Lett. 2019, 21, 3067-3071. doi:10.1021/acs.orglett.9b00652

42. Wu, Y.-C.; Jhong, Y.; Lin, H.-J.; Swain, S. P.; Tsai, H.-H. G.; Hou, D.-R. Adv. Synth. Catal. 2019, 361, 4966-4982. doi:10.1002/adsc.201900997

43. An, Z.; Chen, L.; Jiang, Y.; He, J. Catalysts 2019, 9, 713-724. doi:10.3390/catal9090713

44. Mahe, O.; Dez, I.; Levacher, V.; Briere, J.-F. Angew. Chem., Int. Ed. 2010, 49, 7072-7075. doi:10.1002/anie.201002485

45. Wang, L.; Shirakawa, S.; Maruoka, K. Angew. Chem. 2011, 123, 5439-5442. doi:10.1002/ange.201101307 Angew. Chem. Int. Ed., 2011, 50, 5327-5330.

46. Guo, J.; Yu, S. Org. Biomol. Chem. 2015, 13, 1179-1186. doi:10.1039/c4ob02227k

47. Lebrun, S.; Sallio, R.; Dubois, M.; Agbossou-Niedercorn, F.; Deniau, E.; Michon, C. Eur. J. Org. Chem. 2015, 1995-2004. doi:10.1002/ejoc.201403573

48. Sallio, R.; Lebrun, S.; Capet, F.; Agbossou-Niedercorn, F.; Michon, C.; Deniau, E. Beilstein J. Org. Chem. 2018, 14, 593-602. doi:10.3762/bjoc.14.46

49. Yu, X. H.; Wang, W. Chem. - Asian J. 2008, 3, 516-532. doi:10.1002/asia.200700415

50. Wang, X.-F.; An, J.; Zhang, X.-X.; Tan, F.; Chen, J.-R.; Xiao, W.-J. Org. Lett. 2011, 13, 808-811. doi:10.1021/ol1031188

51. Torán, R.; Vila, C.; Sanz-Marco, A.; Muñoz, M. C.; Pedro, J. R.; Blay, G. Eur. J. Org. Chem. 2020, 627-630. doi:10.1002/ejoc.201901907

52. Hayama, N.; Azuma, T.; Kobayashi, Y.; Takemoto, Y. Chem. Pharm. Bull. 2016, 64, 704-717. doi:10.1248/cpb.c15-00983

53. Michigami, K.; Murakami, H.; Nakamura, T.; Hayama, N.; Takemoto, Y. Org. Biomol. Chem. 2019, 17, 2331-2335. doi:10.1039/c9ob00045c

54. Miyaji, R.; Asano, K.; Matsubara, S. Org. Lett. 2013, 15, 3658-3661. doi:10.1021/ol401538b
55. Liu, X.; Lu, Y. Org. Biomol. Chem. 2010, 8, 4063-4065. doi:10.1039/c0ob00223b

56. Du, J.-Y.; Zeng, C.; Han, X.-J.; Qu, H.; Zhao, X.-H.; An, X.-T.; Fan, C.-A. J. Am. Chem. Soc. 2015, 137, 4267-4273. doi:10.1021/jacs.5b01926

57. Sunoj, R. B. Acc. Chem. Res. 2016, 49, 1019-1028. doi:10.1021/acs.accounts.6b00053

58. Rueping, M.; Kuenkel, A.; Atodiresei, I. Chem. Soc. Rev. 2011, 40, 4539-4549. doi:10.1039/c1cs15087a

59. Saito, K.; Moriya, Y.; Akiyama, T. Org. Lett. 2015, 17, 3202-3205. doi:10.1021/acs.orglett.5b01654

60. Yang, L.; Xia, C.; Huang, H. Chin. J. Catal. 2011, 32, 1573-1576. doi:10.1016/s1872-2067(10)60261-6

61. Feng, Z.; Xu, Q.-L.; Dai, L.-X.; You, S. L. Heterocycles 2010, 80, 765-771. doi:10.3987/com-09-s(s)66

62. Ojha, M.; Bansal, R. K. Curr. Catal. 2021, in press. doi:10.2174/2211544709999201201120617

63. Wang, L.; Chen, J.; Huang, Y. Angew. Chem., Int. Ed. 2015, 54, 15414-15418. doi:10.1002/anie.201508371

64. Mukherjee, S.; Yang, J. W.; Hoffmann, S.; List, B. Chem. Rev. 2007, 107, 5471-5569. doi:10.1021/cr0684016

65. Lee, S.-J.; Youn, S.-H.; Cho, C.-W. Org. Biomol. Chem. 2011, 9, 7734-7741. doi:10.1039/c1ob06078c

66. Guo, H.-M.; Yuan, T.-F.; Niu, H.-Y.; Liu, J.-Y.; Mao, R.-Z.; Li, D.-Y.; Qu, G.-R. Chem. - Eur. J. 2011, 17, 4095-4098. doi:10.1002/chem.201100435

67. Joie, C.; Deckers, K.; Raabe, G.; Enders, D. Synthesis 2014, 1539-1546. doi:10.1055/s-0033-1340982

68. Zhang, J.; Xu, Y.; Wang, Z.; Zhong, R.; Wang, Y. J. Org. Chem. 2021, 86, 4262-4273. doi:10.1021/acs.joc.1c00163

69. Xu, C.-J.; Du, W.; Albrecht, Ł.; Chen, Y.-C. Synthesis 2020, 2650-2661. doi:10.1055/s-0040-1707176

\section{License and Terms}

This is an Open Access article under the terms of the Creative Commons Attribution License (https://creativecommons.org/licenses/by/4.0). Please note that the reuse, redistribution and reproduction in particular requires that the author(s) and source are credited and that individual graphics may be subject to special legal provisions.

The license is subject to the Beilstein Journal of Organic Chemistry terms and conditions:

(https://www.beilstein-journals.org/bjoc/terms)

The definitive version of this article is the electronic one which can be found at: $\underline{\text { https://doi.org/10.3762/bjoc.17.173 }}$ 